QMW-97-16

NI97028-NQF

hep-th/9705162

May, 1997

\title{
Gravitational Duality, Branes and Charges
}

\author{
C.M. Hull \\ Physics Department, Queen Mary and Westfield College, \\ Mile End Road, London E1 4NS, U.K. \\ and \\ Isaac Newton Institute, 20 Clarkson Road, \\ Cambridge CBз OEH, U.K.
}

\begin{abstract}
It is argued that $D=10$ type II strings and M-theory in $D=11$ have $D-5$ branes and 9-branes that are not standard p-branes coupled to anti-symmetric tensors. The global charges in a $D$-dimensional theory of gravity consist of a momentum $P_{M}$ and a dual $D-5$ form charge $K_{M_{1} \ldots M_{D-5}}$, which is related to the NUT charge. On dimensional reduction, $P$ gives the electric charge and $K$ the magnetic charge of the graviphoton. The charge $K$ is constructed and shown to occur in the superalgebra and BPS bounds in $D \geq 5$, and leads to a NUT-charge modification of the BPS bound in $D=4 . K$ is carried by Kaluza-Klein monopoles, which can be regarded as $D-5$ branes. Supersymmetry and U-duality imply that the type IIB theory has $(p, q)$ 9-branes. Orientifolding with $32(0,1)$ 9-branes gives the type I string, while modding out by a related discrete symmetry with 32 (1,0) 9-branes gives the $S O(32)$ heterotic string. Symmetry enhancement, the effective world-volume theories and the possibility of a twelve dimensional origin are discussed.
\end{abstract}




\section{Introduction}

BPS states have played a vital role in the unravelling of the non-perturbative structure of superstring theory and $M$-theory [1-13]. As these break some fraction of the supersymmetry, they carry some charge that appears on the right hand side of the global supersymmetry algebra. The standard example of such a charge is the electric or magnetic charge for some $n$-form anti-symmetric tensor gauge field. This can be a central charge, as in the case of the electric charge for a vector gauge field, but in general it is a $p$-form charge, and the standard example of a BPS state carrying a $p$-form charge is a $p$-brane - an extended object whose world-volume is a $p+1$ dimensional space of Lorentzian signature. For an $n$-form anti-symmetric tensor gauge field in $D$ dimensions, the electric charge is carried by a $p$-brane with $p=n-1$, while the magnetic charge is is carried by a $\tilde{p}$-brane with $\tilde{p}=D-n-3$.

The purpose of this paper is to re-examine the spectrum of BPS branes in string theory and M-theory. In section 2, the charges that occur in the corresponding supersymmetry algebras are studied. In addition to the expected $p$-brane charges, there is an extra $D-5$ form charge $K$ in $D$ dimensions, and an extra 9-form charge for the type IIB theory and for type IIA or M-theory.

The $D-5$ form charge is carried by Kaluza-Klein (KK) monopole space-times [14]. For example, the Kaluza-Klein monopole space-time $N_{4} \times \mathbb{R}^{D-5,1}$, where $N_{4}$ is Euclidean Taub-NUT space, can be thought of as a $D-5$ brane and carries a $D-5$ form charge proportional to the volume form of $\mathbb{R}^{D-5}$. On dimensional reduction to $D-1$ dimensions, the $\mathrm{KK}$ monopole gives rise to magnetic charge of the graviphoton, which in $D-1$ dimensions is a $(D-1)-4$ form charge $Z_{D-5}$. On reduction, the $D-5$ form $K_{D-5}$ gives the magnetic charge $Z_{D-5}$ and the $K$-charge for $D-1$ dimensions, which is a $(D-1)-5$ form charge.

Thus in $D$ dimensions, gravity has two global charges, the ADM momentum $P_{M}$ and the new charge $K_{M_{1} \ldots M_{D-5}}$ which are 'dual' in the same way that electric and magnetic charges are dual in Maxwell theory. Indeed, on dimensional reduction, $P_{M}$ gives the electric charges and $K_{M_{1} \ldots M_{D-5}}$ gives the magnetic charges of 
the graviphotons. Kaluza-Klein monopole space-times $N_{4} \times \mathbb{R}^{D-5,1}$ can be thought of as $D-5$ branes occurring in the purely gravitational sector of the theory; this was suggested in [2], where it was shown that the spectrum of compactified string theories included BPS states arising from wrapping such $D-5$ branes. In section 3, the charge $K_{M_{1} \ldots M_{D-5}}$ is constructed explicitly and is shown to occur in superalgebras evaluated in suitable backgrounds. The K-charge is related to the NUT charge, and the supersymmetry-motivated formula for $K$ gives a new expression for the NUT charge, which can be used to prove the generalisation of the gravitational Bolgomolnyi bound [15] due to NUT charge; this is done in section 4. In section 5 , the charge $K$ is calculated for certain examples. The interpretation of $N_{4} \times \mathbb{R}^{D-5,1}$ as a $D-5$ brane would suggest that its collective coordinates should form a $D-4$ dimensional supermultiplet including 4 scalars, corresponding to the position of the brane in $N_{4}$. However, no such multiplet exists in e.g. 7 dimensions (corresponding to $D=11$ ). This problem is addressed and resolved in section 8 , where the collective coordinate structure is found. There is a sense in which the KK monopole can be regarded as a 'twisted' $D-4$ brane [2], and the number of translational zero modes is that appropriate for a $D-4$ brane.

The configurations that preserve half the supersymmetry include pp-wave configurations in addition to the $p$-branes and KK monopoles. On compactification, the set of all BPS states in the compactified theory includes $p$-branes and $\mathrm{KK}$ monopoles wrapped around internal dimensions, together with pp-waves moving in the compactifying space [2]. These are all on an equal footing in the compactified theory, and related by U-duality [2]; in particular, pp-waves in an internal direction are related to fundamental strings by T-duality [16]. There is then a sense in which pp-waves are a type of string, so that pp-waves and KK monopoles can be thought of as gravitational branes, or 'G-branes'. The pp-waves carry the charge $P$ and KK monopoles carry the dual charge $K$, and on compactification these G-branes are U-dual to $p$-branes which couple to anti-symmetric tensor gauge fields [2].

The type IIB theory has $p$-branes for $p=1,3,5,7,9$ and in particular has a Dirichlet 9-brane, which gives rise to Chan-Paton factors for open strings. The 
IIB superalgebra implies that the $p$-form charges carried by $p$-branes are singlets under the $S L(2, \mathbb{Z})$ duality symmetry for $p=3,7$ and are doublets for $p=1,5,9$. For $p=1,5$, this is in accord with the existence of $(p, q)$ strings and $(p, q) 5$-branes [25], with charge $p$ with respect to the NS-NS (Neveu-Schwarz Neveu-Schwarz) 2form and charge $q$ with respect to the RR (Ramond-Ramond) 2-form. The algebra suggests that there should also be $(p, q)$ 9-branes. Indeed, acting on the Dirichlet 9-brane with $S L(2, \mathbb{Z})$ must either leave the brane invariant, or give a new 9-brane, and the superalgebra implies that the 9-brane charge must transform, so that the action of $S L(2, \mathbb{Z})$ on the Dirichlet 9-brane generates $(p, q)$ 9-branes.

Of course, this is rather formal as it is not consistent to have Dirichlet 9branes in general backgrounds. However, orientifolding the type IIB string to give the type I string [12], i.e. modding out the by the perturbative $\mathbb{Z}_{2}$ world-sheet parity reversal symmetry $\Omega$, requires the presence of 32 Dirichlet 9-branes, which give rise to $S O(32)$ Chan-Paton factors [13]. This suggests that the new 9-branes might play a role in similar constructions. This is addressed in section 6 , where it is shown that there must be a non-perturbative $\mathbb{Z}_{2}$ symmetry $\tilde{\Omega}$ such that modding out by $\tilde{\Omega}$ in the presence of $32(1,0) 9$-branes gives the $S O(32)$ heterotic string. The strong-weak coupling duality of type IIB interchanges the $(0,1) 9$-branes with the (1,0) 9-branes and $\Omega$ with $\tilde{\Omega}$, so that this is consistent with the duality between type I and $S O(32)$ heterotic strings [4,10-13]. The new (1,0) 9-branes are essential for the construction and there are analogous constructions using $(p, q)$ 9-branes.

In section 7, it is argued using T-duality that the presence of a new $(1,0) 9$-brane in type IIB theory implies the existence of a new 9-brane in the IIA theory, which should originate from a 9-brane in M-theory; in section 2, it is shown that such 9 -form charges can indeed occur in the relevant superalgebras. The possibility of a 9-brane in M-theory has also been discussed in [24,54]. In section 8, the effective world-volume theories describing the collective coordinates for all branes, including G-branes, are found, with supersymmetry implying that the KK monopole has less translational zero-modes than might have been expected naively. All M-theory charges could naturally arise from a 2-form, a self-dual 6-form and a 10-form charge 
in 12 dimensions, but there is no way the effective world-volume theories of all Mbranes can arise from covariant dynamics of branes in twelve dimensions. In section 9, the gauge symmetry enhancement from coincident G-branes is discussed.

With these 'new branes' included, all the charges that can occur on the right hand side of the superalgebra in fact do occur, and are needed to get the right counting on compactification, where the new branes give rise to branes related to known ones by U-duality.

\section{Superalgebras and Brane Charges}

The most general super-Poincaré algebra in $D$ dimensions includes some antisymmetric tensor charges in the anti-commutator of two supercharges, and these commute with all generators except the Lorentz generators. The anti-commutator of two supercharges gives ${ }^{\star}$ a symmetric bi-spinor $Z_{\alpha \beta}$ defined by $\left\{Q_{\alpha}, Q_{\beta}\right\}=Z_{\alpha \beta}$, and $Z_{\alpha \beta}$ can be rewritten as a sum of terms, each of which is of the form $Z_{M N \ldots Q}\left(\Gamma^{M N \ldots Q} C\right)_{\alpha \beta}$ for some set of $p$-form charges $Z_{M_{1} \ldots M_{p}}$. The values of $p$ which occur will depend on the dimension considered, but for a conventional superalgebra will include a 1 -form corresponding to the space-time momentum $P_{M}$. For a theory with $N$ supercharges $Q_{\alpha}^{a}$ with $a=1, \ldots, N$, the extended superalgebra includes the anti-commutator $\left\{Q_{\alpha}^{a}, Q_{\beta}^{b}\right\}=Z_{\alpha \beta}^{a b}+\bar{Z}_{\alpha \beta}^{a b}$ where $Z_{\alpha \beta}^{a b}=Z_{\alpha \beta}^{b a}=Z_{\beta \alpha}^{a b}$ and $\bar{Z}_{\alpha \beta}^{a b}=-\bar{Z}_{\alpha \beta}^{b a}=-\bar{Z}_{\beta \alpha}^{a b}$.

In $D=11$ the $N=1$ superalgebra has one 32-component Majorana supercharge $Q_{\alpha}$ and these satisfy the anti-commutation relations [17,5]

$$
\left\{Q_{\alpha}, Q_{\beta}\right\}=\left(\Gamma^{M} C\right)_{\alpha \beta} P_{M}+\frac{1}{2 !}\left(\Gamma^{M N} C\right)_{\alpha \beta} Z_{M N}+\frac{1}{5 !}\left(\Gamma^{M N P Q R} C\right)_{\alpha \beta} Z_{M N P Q R} .
$$

The left-hand side is symmetric in its spinor indices and so has $32.33 / 2=528$ components in general. Anti-symmetric tensor charges are included on the righthand-side to give a total of $11+55+462=528$ components. Dimensionally reducing

$\star$ The notation and conventions are as in [5]. The vector indices are $M=0, \ldots D-1$ and the spinor indices are $\alpha, \beta$. The charge conjugation matrix is $C_{\alpha \beta}$ and $\Gamma^{M N \ldots P}=\Gamma^{[M} \Gamma^{N} \ldots \Gamma^{P]}$. 
to $D=10$ gives the IIA algebra, with the anti-commutator of two Majorana supercharges being

$$
\begin{aligned}
\left\{Q_{\alpha}, Q_{\beta}\right\}= & \left(\Gamma^{M} C\right)_{\alpha \beta} P_{M}+\left(\Gamma_{11} C\right)_{\alpha \beta} Z+\left(\Gamma^{M} \Gamma_{11} C\right)_{\alpha \beta} Z_{M}+\frac{1}{2 !}\left(\Gamma^{M N} C\right)_{\alpha \beta} Z_{M N} \\
& +\frac{1}{4 !}\left(\Gamma^{M N P Q} \Gamma_{11} C\right)_{\alpha \beta} Z_{M N P Q}+\frac{1}{5 !}\left(\Gamma^{M N P Q R} C\right)_{\alpha \beta} Z_{M N P Q R}
\end{aligned}
$$

The IIB supersymmetry algebra has two Majorana-Weyl supercharges, $Q_{\alpha}^{a}$, $(a=1,2)$, of the same chirality with

$$
\begin{aligned}
\left\{Q_{\alpha}^{a}, Q_{\beta}^{b}\right\} & =\delta^{a b}\left(\mathcal{P} \Gamma^{M} C\right)_{\alpha \beta} P_{M}+\left(\mathcal{P} \Gamma^{M} C\right)_{\alpha \beta} Z_{M}^{a b}+\frac{1}{3 !} \varepsilon^{a b}\left(\mathcal{P} \Gamma^{M N P} C\right)_{\alpha \beta} Z_{M N P} \\
& +\frac{1}{5 !} \delta^{a b}\left(\mathcal{P} \Gamma^{M N P Q R} C\right)_{\alpha \beta}\left(Z^{+}\right)_{M N P Q R} \\
& +\frac{1}{5 !}\left(\mathcal{P} \Gamma^{M N P Q R} C\right)_{\alpha \beta}\left(Z^{+}\right)_{M N P Q R}^{a b}
\end{aligned}
$$

where $Z_{M}^{a b}$ and $\left(Z^{+}\right)_{M N P Q R}^{a b}$ are $2 \times 2$ traceless symmetric matrices in $a, b, \delta_{a b} Z_{M}^{a b}=$ 0 and $\delta_{a b}\left(Z^{+}\right)_{M N P Q R}^{a b}=0$, and $\mathcal{P}=\frac{1}{2}\left(1+\Gamma^{11}\right)$ is the chiral projector. All three of the 5-form charges $\left(Z^{+}\right)_{M N P Q R},\left(Z^{+}\right)_{M N P Q R}^{a b}$ are self-dual 5-forms, $* Z^{+}=Z^{+}$. The total number of components of all charges on the RHS of $(2.3)$ is $10+2 \times 10+$ $120+126+2 \times 126=528$, which again balances the number of components on the LHS.

The $P_{M}$ on the RHS of (2.1)-(2.3) is the $D$-momentum while some of the $p$-form charges $Z_{M_{1} \ldots M_{p}}$ are carried by $p$-branes. An $n+1$-form field strength $F_{M_{1} \ldots M_{n+1}}$ satisfies the field equations

$$
\nabla_{N}(* \tilde{F})^{N M_{1} \ldots M_{n}}=J^{M_{1} \ldots M_{n}}
$$

and

$$
\nabla_{N}(* F)^{N M_{1} \ldots M_{\tilde{n}}}=\tilde{J}^{M_{1} \ldots M_{\tilde{n}}}
$$

where $\tilde{n}=D-n-2$, and $\tilde{F}$ is given by varying the action $S$ with respect to $F$ :

$$
\tilde{F}^{M_{1} \ldots M_{\tilde{n}+1}} \equiv \frac{1}{(n+1) !} \varepsilon^{M_{1} \ldots M_{\tilde{n}+1} N_{1} \ldots N_{n+1}} \frac{\delta S}{\delta F_{N_{1} \ldots N_{n+1}}}=* F^{M_{1} \ldots M_{\tilde{n}+1}}+\ldots
$$


so that $\tilde{F}=* F+\ldots$ where $* F$ is the Hodge dual, and the dots denote terms dependent on the interactions; for the free theory, $\tilde{F}=* F$. The currents $J, \tilde{J}$ satisfy the conservation laws

$$
\nabla_{M_{1}} J^{M_{1} \ldots M_{n}}=0, \quad \nabla_{M_{1}} \tilde{J}^{M_{1} \ldots M_{\tilde{n}}}=0
$$

In regions in which the magnetic source $\tilde{J}$ vanishes, $F$ can be written in terms of an $n$-form potential $A, F=d A$, while in regions in which the electric source $J$ vanishes, $\tilde{F}$ can be written in terms of a $\tilde{n}$-form potential $\tilde{A}, \tilde{F}=d \tilde{A}$.

An electric $p$-brane source couples to a $n=p+1$ form potential $A$ through the Wess-Zumino term *

$$
\int d^{n} \sigma A_{M_{1} \ldots M_{n}} \epsilon^{m_{1} \ldots m_{n}} \partial_{m_{1}} X^{M_{1}} \ldots \partial_{m_{n}} X^{M_{n}}
$$

where $\sigma^{m}$ are world-volume coordinates, $m=1, \ldots, n=p+1$, and $X^{M}(\sigma)$ is the position of the brane. This then leads to an $n$-form current density

$$
J^{M_{1} \ldots M_{n}}(X)=\int d^{n} \sigma \epsilon^{m_{1} \ldots m_{n}} \partial_{m_{1}} X^{M_{1}} \ldots \partial_{m_{n}} X^{M_{n}} \delta\left(X^{M}-X^{M}(\sigma)\right)
$$

A magnetic $p=\tilde{n}-1$ brane source couples to $\tilde{A}$ and leads to a similar formula for $\tilde{J}$, with $n$ replaced by $\tilde{n}$. A static configuration carries an electric charge density $z_{i_{1} \ldots i_{p}}=J_{0 i_{1} \ldots i_{p}}$ and a magnetic charge density $\tilde{z}_{i_{1} \ldots i_{\tilde{p}}}=\tilde{J}_{0 i_{1} \ldots i_{\tilde{p}}}$ where $n=p+1$, $\tilde{n}=\tilde{p}+1$ and $i, j=1, \ldots D-1$ are spatial indices. These charge densities give rise to total charges $Z_{i_{1} \ldots i_{p}}, \tilde{Z}_{i_{1} \ldots i_{\tilde{p}}}$ which are again anti-symmetric tensors carrying purely spatial indices.

These $\delta$-function sources can often be replaced by solitonic $p$-brane solutions of the theory [18-24]. A spatial slice of a $p$-brane configuration has an asymptotic 'boundary' at spatial infinity given by $\mathbb{R}^{p} \times S^{D-p-2}$ for an infinite brane, or by

\footnotetext{
$\star$ The alternating symbol $\epsilon^{M_{1}} \ldots M_{\tilde{n}+1}$ is a tensor density while $\varepsilon^{M_{1} \ldots M_{\tilde{n}+1}}$ is a tensor.
} 
$\mathbb{R}^{p-q} \times T^{q} \times S^{D-p-2}$ if the $p$-brane is wrapped around $q$ toroidal dimensions. The charge of an electric $p$-brane coupled to a $n=p+1$ form potential $A$ and aligned in the directions given by the spatial $p$-form $v$ is given by

$$
Z . v \equiv \frac{1}{p !} Z_{i_{1} \ldots i_{p}} v^{i_{1} \ldots i_{p}}=\frac{1}{\Omega_{\tilde{n}+1}} \int_{S^{\tilde{n}+1}} \tilde{F}
$$

where the integral is over the $\tilde{n}+1$ sphere at transverse spatial infinity surrounding the brane and $\Omega_{d}$ is the area of the unit $d$-sphere. Similarly, the charge of a magnetic $p$-brane coupled to an $\tilde{n}$-form potential $A$ with $\tilde{n}=D-n-2=p+1$ is

$$
\tilde{Z} . v \equiv \frac{1}{p !} \tilde{Z}_{i_{1} \ldots i_{p}} v^{i_{1} \ldots i_{p}}=\frac{1}{\Omega_{n+1}} \int_{S^{n+1}} F
$$

where the integral is over the $n+1$ sphere at transverse spatial infinity surrounding the brane.

A $p$-brane carries a $p$-form charge $Z_{i_{1} \ldots i_{p}}$ with purely spatial indices. The spatial components of the $Z_{M_{1} . . M_{p}}$ appearing in the algebras (2.1)-(2.3) can arise as $p$ brane charges, but this leaves the question of the interpretation of the components $Z_{0 i_{1} \ldots i_{q}}$. These can be dualised to a spatial $\hat{q}$-form charge $\hat{Z}_{i_{1} \ldots i_{\hat{q}}}$ given by the spatial components of $* Z$,

$$
\hat{Z}_{j_{1} \ldots j_{\hat{q}}}=\frac{1}{(q+1) !} \varepsilon_{j_{1} \ldots j_{\hat{q}} 0 i_{1} \ldots i_{q}} Z^{0 i_{1} \ldots i_{q}}
$$

which could arise as a $\hat{q}=D-q-1$ brane charge, and this is the most natural interpretation of the charge $Z_{0 i_{1} \ldots i_{q}}{ }^{\dagger}$ We can now compare this with the expected $p$-brane spectrum for M-theory and type II strings.

Consider first $D=11$; M-theory has a membrane and a 5 -brane coupling to the 3 -form gauge field. The algebra (2.1) has a 2-form $Z_{M N}$ charge which gives a 2brane charge $Z_{i j}$ and a 9-brane charge $\hat{Z}_{i_{1} \ldots i_{9}}$, and a 5-form charge $Z_{M N P Q R}$, which

$\dagger$ This interpretation has been suggested independently by Paul Townsend. 
gives a 5 -brane charge $Z_{i j k l m}$ and a 6 -brane charge $\hat{Z}_{i_{1} \ldots i_{6}}$. Thus the superalgebra has charges that could correspond to an extra 6-brane and an extra 9-brane, so that the question arises as to whether these actually occur and, if so, what is their interpretation.

For the type IIA theory, the expected branes are a string and a 5-brane, together with D-branes for $p=0,2,4,6,8$. The central charge $Z$ in $(2.2)$ is the 0-brane charge, the 4-form charge $Z_{M N P Q}$ gives a 4-brane charge $Z_{i j k l}$ and a 6 brane charge $\hat{Z}_{i j k l m n}$, while the 2 -form charge $Z_{M N}$ gives a 2-brane charge $Z_{i j}$ and an 8-brane charge $\hat{Z}_{i j k l m n p q}$. The 5 -form charge $Z_{M N P Q R}$ gives two 5 -brane charges, $Z_{i j k l m}$ and $\hat{Z}_{i j k l m}$, while the 1 -form charge $Z_{M}$ gives a string charge $Z_{i}$ and a 9-brane charge $\hat{Z}_{i_{1} \ldots i_{9}}$. Comparing with the expected brane scan, we are finding charges that could correspond to an extra 5-brane and an extra 9-brane.

Finally, for the type IIB string, the expected branes are a string and a 5-brane, together with D-branes for $p=1,3,5,7,9$ carrying Ramond-Ramond charge. There are also $(p, q)$ strings carrying NS-NS string charge $p$ and RR string charge $q$. In the quantum theory, $q, p$ are integers and non-trivial bound states occur whenever $p$ and $q$ are co-prime [25]. Similarly, there are $(p, q)$ 5-branes, and for both strings and 5-branes the 2-vector of charges $(p, q)$ transforms as a doublet under the action of $S L(2, \mathbb{Z})$ U-duality transformations. In the algebra (2.3), the the 3-form charge $Z_{M N P}$ gives a 3 -brane charge $Z_{i j k}$ and a 7 -brane charge $\hat{Z}_{i j k l m n p}$. The two 1 -form charges $Z_{M}^{a b}$ give two string charges $Z_{i}^{a b}$ and two 9-brane charges $\hat{Z}_{i_{1} \ldots i_{9}}^{a b}$. A general 5-form charge $Z_{M N P Q R}$ gives two 5 -brane charges, $Z_{i j k l m}$ and $\hat{Z}_{i j k l m}$, but for a self-dual 5-form charge, these are equal, $Z_{i j k l m}=\hat{Z}_{i j k l m}$. Thus the three selfdual 5-form charges $\left(Z^{+}\right)_{M N P Q R},\left(Z^{+}\right)_{M N P Q R}^{a b}$ give the charges for 3 five-branes, $\left(Z^{+}\right)_{i j k l m},\left(Z^{+}\right)_{i j k l m}^{a b}$

The $S L(2, \mathbb{R})$ symmetry of the supergravity equations of motion acts through a compensating $S O(2)$ transformation in symmetric gauge, so that the index $a=1,2$ on the supercharges undergoes $S O(2)$ rotations. The two charges $Z_{i}^{a b}$ are a doublet of $S O(2)$ and arise from charges which are a doublet of $S L(2)$ (see section 6), and 
correspond to the charges of $(p, q)$ strings. Similarly, the two charges $\left(Z^{+}\right)_{i j k l m}^{a b}$ correspond to the charges of $(p, q) 5$-branes. Similarly, the two 9-brane charges $\hat{Z}_{i_{1} \ldots i_{9}}^{a b}$ could correspond to the charges of $(p, q)$ 9-branes. Note that the 3 -brane and 7-brane charges are singlets under the action of $S O(2)$. This was expected for the 3-brane since the 4-form gauge field to which it couples does not transform under $S L(2, \mathbb{R})$. The 7 -brane couples to the axion which does transform under $S L(2, \mathbb{R})$. Acting on the 7 -brane solutions of $[23]$ with $S L(2, \mathbb{Z})$ gives a new 7brane with the same 7-brane charge (the coupling to the 8-form potential which is dual to the axion is unchanged) but the $S L(2, \mathbb{Z})$ monodromy changes and the couplings to other branes also changes [26]. In the IIB algebra, there are thus charges for an extra 5-brane and for $(p, q)$ 9-branes.

We now turn to the interpretation of the extra charges that arise in the above cases. We have seen that in $D=10,11$ dimensions, there is an extra spatial $(D-5)$-form charge that does not seem to have an interpretation in terms of electric or magnetic $p$-brane charges - we have already accounted for all of these. In the following sections, it will be seen that this $(D-5)$-form charge is non-zero for Kaluza Klein monopole space-times in which all the anti-symmetric tensor gauge fields vanish. It will be argued that in any theory of gravity in $D>4$ dimensions, there is, in addition to the ADM momentum $P_{M}$, a $(D-5)$-form charge $K_{M_{1} \ldots M_{D-5}}$. This gives rise to the extra 6-brane charge in $D=11$ and the extra 5-brane charge in both type II theories in $D=10$. The algebras also suggest that there should be a 9-brane in $D=11$ and a 9-brane in both the IIA and IIB theories. These extra 9-branes can be formally thought of as coupling to a non-dynamical 10-form potential, which could arise as an auxiliary field that is analogous to the 4-form in four-dimensional supergravity which couples to 3-branes that was discussed in [27]. In the IIB theory, the 10-form to which the Dirichlet 9-brane couples is associated with the RR sector, suggesting that the new (1,0) 9-branes in the IIA and IIB theory be associated with the NS-NS sector. The $D=119$-brane gives rise to the 8-brane and the 9-brane of the IIA theory, while the NS-NS and the RR 9-branes of the type IIB theory combine to form $(p, q)$ 9-branes, with the $S L(2, \mathbb{Z})$ U-duality 
acting on the $(p, q)$ 9-brane charge lattice. This will be discussed further in section 6.

If the $D$-momentum $P_{M}$ were treated in the same way as the other charges, it would be split into the spatial momentum $P_{i}$ which is analogous to a 1-brane charge, and the energy $P_{0}$, which could be dualised to a $D-1$ brane charge $\hat{P}_{i_{1} \ldots i_{D-1}}$. The fact that states carrying momentum in compactified dimensions are U-dual to states obtained by wrapping branes round the compactified dimensions [2] means that for some purposes states carrying the gravitational charge $P_{M}$ should be treated on the same footing as states carrying brane charges. The BPS states associated with the charge $P_{i}$ are pp-waves moving in the $i$ th dimension with momentum $P_{i}$ and, as discussed in the introduction, these can be regarded as strings or branes for some purposes. Indeed, if the $i^{\prime}$ th dimension is compact, the pp-wave with momentum $P_{i}=n_{i}$ say is T-dual to a string winding around the $i$ 'th dimension with winding number or string charge $Z_{i}=n_{i}[16]$. The fact that the energy appears to be associated with a $D-1$ brane seems natural from a 12-dimensional perspective, as will be discussed in section 7 .

\section{Magnetic Charges in Gravity}

We have seen that the IIA,IIB and $D=11$ superalgebras have an extra $(D-5)$-form charge $K_{i_{1} \ldots i_{D-5}}$ that it would be natural to associate with a $D-5$ brane. On dimensionally reducing, the $D=116$-form charge gives a 6 -form charge and a 5 -form charge in $D=10$. The 6 -form charge is the magnetic charge for the RR vector field of the type IIA theory, which comes from the $g_{\mu 11}$ components of the $D=11$ metric (where the reduction is in the $x^{11}$ direction). Thus the $D=106$-form charge is the magnetic charge of the graviphoton, which arises from Kaluza-Klein monopole solutions of the $D=11$ theory [3], so that the new 6-form charge in $D=11$ should be non-trivial in Kaluza-Klein monopole space-times. The standard Kaluza-Klein monopole space-time in $D$ dimensions is $N_{4} \times \mathbb{R}^{D-5,1}$ where $N_{4}$ is 4-dimensional self-dual Euclidean Taub-NUT space (or its multi-centre 
generalisation [28]) and $\mathbb{R}^{D-5,1}$ is $D-4$ dimensional Minkowski space. This is a solution of string theory with $D=10$ and of $M$-theory with $D=11$, since selfdual (multi-)Taub-NUT space is hyperkahler and so is the target space of a $(4,4)$ superconformal sigma-model for which the beta-functions vanish [29]. The NUT charge $[30,31,45]$ of the Taub-NUT space is related to the magnetic charge of the graviphoton on dimensionally reducing [14], so that we learn that the 6-form charge in $D=11$ is related to NUT charge. However, the NUT charge is defined for the 4 dimensional space $N_{4}$, and can be readily generalised to $D \geq 4$ dimensions, while the $D-5$ form charge $K$ only exists in $D \geq 5$ dimensions, so that it cannot be precisely the $D$-dimensional generalisation of NUT charge, although it is closely related to this.

Consider the Kaluza-Klein reduction of gravity from $D$ to $D-1$ dimensions. The $D$-dimensional metric $g_{M N}$ gives a metric $g_{\mu \nu}$, a vector field $A_{\mu}$ and a scalar $\phi$ in $D-1$ dimensions. The $D$-momentum reduces to the $D-1$ momentum $P_{\mu}$ and the electric charge $Q$. In $D-1$ dimensions, the magnetic charge for a Maxwell field $A_{\mu}$ is a $(D-1)-4=D-5$ form, which should have a $D$-dimensional origin. Postulating a charge $K_{i_{1} \ldots i_{D-5}}$ in $D$ dimensions, the reduction to $D-1$ dimensions then gives a $D-5$ form which can be associated with the magnetic charge of the graviphoton $A_{\mu}$ and a $D-6$ form $K_{m_{1} \ldots m_{D-6}}$ (where $m=1, \ldots, D-2$ labels the spatial dimensions in $D-1$ dimensions) which corresponds to the K-charge $(D-1)-5$ form in $D-1$ dimensions. Thus the gravitational charges $P_{1}, K_{D-5}$ in $D$ dimensions give the gravitational charges $P_{1}, K_{(D-1)-5}$ in $D-1$ dimensions, together with the electric and magnetic charges $Q, Z_{(D-1)-4}$ for the graviphoton $A_{1}$ (where the subscript denotes the degree of the form).

We now turn to the definition of the gravitational charges $P, K$. We shall be interested in $D$-dimensional space-times $M$ for which a spacelike slice is bounded by a $D-2$ dimensional 'surface at infinity', $\Sigma_{D-2}$. For asymptotically flat spaces, this is a $D-2$ sphere, $\Sigma_{D-2}=S^{D-2}$, while for toroidally wrapped $p$-brane spacetimes it is $\Sigma_{D-2}=T^{p} \times S^{D-p-2}$. In particular, for a $D-4$ brane it is $\Sigma_{D-2}=$ $T^{D-4} \times S^{2}$. For KK monopole space-times, it is a twisted version of these $D-4$ 
brane asymptotics, in which one of the toroidal directions is a fibre for a circle bundle over $S^{2}$. For a single monopole, this gives the Hopf fibration of $S^{3}$ as a circle bundle over $S^{2}$, so that topologically $\Sigma_{D-2}=T^{D-5} \times S^{3}$. The $S^{3}$ has a squashed metric - as a radial coordinate $r \rightarrow \infty$, the size of the Hopf fibres tends to a constant while the area of the 2 -sphere increases as $r^{2}$. For the $s$ monopole generalisation, the surface at infinity is $\Sigma_{D-2}=T^{D-5} \times \tilde{\Sigma}_{3}$ where $\tilde{\Sigma}_{3}$ is the (squashed) 3 -sphere for $s=1$ or a (squashed) Lens space $L(s, 1)=S^{3} / \mathbb{Z}_{s}$ for $s>1$. (The Lens space $L(s, 1)$ is defined by regarding $S^{3}$ as the points $(z, w)$ in $\mathbb{C}^{2}$ with $|z|^{2}+|w|^{2}=1$ and identifying $(z, w)$ with $\left(e^{2 \pi i / s} z, e^{2 \pi i / s} w\right)$.) We shall for now suppose that $\Sigma_{D-2}$ is compact, but later we will consider the limit in which the torus $T^{p}$ becomes large, so that $\Sigma_{D-2}$ tends to $\mathbb{R}^{p} \times S^{D-p-2}$; in this latter case it will become necessary to define the charge densities per unit $p$-volume instead of the total charges, which become infinite.

The KK monopole space $N_{4} \times T^{D-5} \times \mathbb{R}$ can be thought of as a wrapped $D-5$ brane or as a twisted form of a $D-4$ brane, as $N_{4}$ (with a set of fixed points or NUTs removed) has the structure of a circle bundle over a base $\Sigma$, so that locally it has the wrapped $D-4$ brane structure $\Sigma \times T^{D-4} \times \mathbb{R}$. Indeed, as we shall see, it has a $D-5$ form charge, which is usually associated with a $D-5$ brane, whereas it has the number of translational zero modes appropriate for a $D-4$ brane, as will be seen in section 8 . On dimensionally reducing with respect to the toroidal directions, the charges in the resulting $D-p$ dimensional space correspond to charge densities per unit $p$-volume. For the KK monopole, the Hopf fibre cannot become non-compact without introducing irremovable Dirac string singularties in the metric, although the connection and curvature remain well-defined. (It was suggested in [31] that such singularities might be acceptable for some purposes, and it would be interesting to explore their consequences for string theory.) For KK monopoles, one can divide by the length of the Hopf fibre to define densities, as for a $D-4$ brane.

Suppose that there is an asymptotic region in which the space-time $\left(M, g_{M N}\right)$ becomes close to a background space-time $\left(\bar{M}, \bar{g}_{M N}\right)$, with $g_{M N}=\bar{g}_{M N}+h_{M N}$ 
with $h_{M N}$ asymptotically small. In the case of $p$-brane space-times, we shall consider the case in which $\bar{M}$ is $\mathbb{R}^{D-p-1,1} \times T^{p}$ with a flat metric $\bar{g}_{M N}$, with $h_{M N}$ falling off as $O\left(r^{D-3}\right)$ as $r \rightarrow \infty$, where $r$ is a radial coordinate on $\mathbb{R}^{D-p-1}$, so that for large $r, r=$ constant defines a $D-p-2$ sphere of area $r^{D-p-2} \Omega_{D-p-2}$, where $\Omega_{D-p-2}$ is the area of the unit $D-p-2$ sphere.

For the KK monopole, the question of boundary conditions is more subtle. We can take $\bar{M}=\mathbb{R}^{4,1} \times T^{D-5}$ as Taub-NUT is topologically $\mathbb{R}^{4}$, while for the multi-monopole configurations $\mathbb{R}^{4}$ is replaced by $\mathbb{R}^{4} / \mathbb{Z}_{s}$. (For $s>1$, it is sometimes convenient to work with the covering space $\mathbb{R}^{4}$ instead of $\mathbb{R}^{4} / \mathbb{Z}_{s}$, and factor by $\mathbb{Z}_{s}$ at the end.) There are two approaches to choosing the background metric $\bar{g}_{M N}$. One is to fix a topological class of metrics, and consider only metrics within that class. The natural choice of background in this case is $\bar{N}_{4} \times T^{D-5} \times \mathbb{R}$ where $\bar{N}_{4}$ is selfdual Taub-NUT space or the $s$-centre self-dual generalisation, and to consider the class of spaces asymptotic to this, with the boundary surface at space-like infinity given by $\Sigma_{D-2}=T^{D-5} \times S^{3} / \mathbb{Z}_{s}$. On dimensional reduction, this corresponds to fixing the magnetic charge, and considering only spaces with that magnetic charge. In discussing electromagnetism, however, it is more convenient for some purposes to allow all possible magnetic charges, even though these correspond to topologically distinct $U(1)$ bundles, and compare these to the vacuum which is the trivial bundle with zero magnetic charge. Here, this corresponds to allowing boundary conditions with $\Sigma_{D-2}=T^{D-5} \times S^{3} / \mathbb{Z}_{s}$ for all values of $s$, and comparing with the flat background metric $\bar{g}_{M N}$ of the Kaluza-Klein vacuum $T^{D-4} \times \mathbb{R}^{3,1}$, with boundary at space-like infinity given by $\Sigma_{D-2}=T^{D-4} \times S^{2}$. In much of what follows, either choice of boundary conditions could be used, and the formulae give the difference between the charges $P, K$ of the space-time $(M, g)$ and the charges $\bar{P}, \bar{K}$ of the background $(\bar{M}, \bar{g})$. However, it will be convenient to present the results with respect to the KK vacuum, for which we take $\bar{P}=0, \bar{K}=0$.

Introducing frames $e_{M}^{A}$ with $e_{M}^{A} e_{N}^{B} \eta_{A B}=g_{M N}$ etc and the corresponding torsion-free spin-connection $\omega_{M} A B$, Nestor's expression for the ADM momentum 
$P_{M}[32]$ can be generalised to $D$-dimensions to give

$$
P[u]=\frac{1}{2 \Omega_{D-p-2}} \int_{\Sigma_{D-2}} u^{[M} e_{B}^{N} e_{C}^{P]} \Delta \omega_{P}{ }^{B C} d \Sigma_{M N}
$$

Here $\Delta \omega_{P}^{B C}=\omega_{P}{ }^{B C}-\bar{\omega}_{P}{ }^{B C}$ is the difference between the connection on $M$ and that on $\bar{M}$ and so is an an asymptotic tensor, so that (3.1) is covariant. The vector $u$ is an asymptotic Killing vector, so that as $r \rightarrow \infty, u$ tends to a Killing vector of $\bar{M}$. Then $P[u]$ is the conserved charge corresponding to $u$. If $\bar{M}$ has translational Killing vectors $k_{M}^{a}$ labelled by some index $a$, then $u_{M}=u_{a} k_{M}^{a}$ for some functions $u_{a}$ tending to constants $\bar{u}_{a}$ as $r \rightarrow \infty$. Then $P[u]$ can be written as $P^{a} \bar{u}_{a}$, defining the ADM $D$-momentum $P^{a}=P\left[k^{a}\right]$. In the simplest cases in which there is asymptotic flatness, the index $a$ can be identified with the index $A$ labelling the asymptotic frames (see [33]). This is equivalent to other forms of the ADM momentum [32] and is properly the difference between the ADM momentum and that of the background. It can be written in form notation as

$$
P[u]=\frac{1}{2 \Omega_{D-2}} \int_{\Sigma_{D-2}} *\left(e_{\wedge}^{A} e_{\wedge}^{B} u\right)_{\wedge} \Delta \omega_{A B}
$$

where $e^{A}=e_{M}^{A} d x^{M}, \Delta \omega_{A B}=\Delta \omega_{M A B} d X^{M}$ and $u=u_{M} d x^{M}$. If $p$ toroidal directions are Killing, so that $\Sigma_{D-2}=\tilde{\Sigma}_{D-p-2} \times T^{p}$, then the momentum is proportional to the volume $V_{p}$ of the $T^{p}$,

$$
P[u]=V_{p} \tilde{P}[u], \quad \tilde{P}[u]=\frac{1}{2 \Omega_{D-p-2}} \int_{\tilde{\Sigma}_{D-p-2}} u^{[M} e_{B}^{N} e_{C}^{P]} \Delta \omega_{P}^{B C} d \Sigma_{M N}
$$

defining the density $\tilde{P}[u]$ which can be written as an integral over the surface $\tilde{\Sigma}_{D-p-2}$ at transverse spatial infinity [34]. Then the density is well-defined in the decompactification limit in which $V_{p} \rightarrow \infty$, defining the density of an infinite brane space-time. This can be generalised to the case in which the toroidal directions correspond to asymptotic Killing vectors, in which case $V_{p}$ is the asymptotic volume of the $p$-torus [34]. 
Consider a $D-5$ brane space-time, so that the surface at infinity is $\Sigma_{D-2}=$ $\tilde{\Sigma}_{3} \times T^{D-5}$, and let $v$ be a $D-5$-form tending asymptotically to the volume form $\bar{v}$ on $T^{D-5}$. As we shall see, calculating the supersymmetry algebra in such a space gives a K-charge in general, and the expression for this charge that arises motivates the following definition. The $D-5$-form charge $K$

$$
K[v]=\frac{1}{5 !} K_{i_{1} \ldots i_{D-5}} v^{i_{1} \ldots i_{D-5}}
$$

is given by

$$
K[v]=\frac{1}{16 \pi^{2}} \int_{\Sigma_{D-2}} \omega_{\wedge} v
$$

where $\omega$ is the 3 -form whose components are the totally antisymmetric part of the spin-connection (minus the background), $\Delta \omega_{[A B C]}$,

$$
\omega=\frac{1}{2} e_{\wedge}^{B} e_{\wedge}^{A} \Delta \omega_{A B}
$$

Again, this is the difference between the K-charge and that of the background, and if the toroidal directions are asymptotically Killing,

$$
K[v]=V_{p} \tilde{K}[v], \quad \tilde{K}[v]=\frac{1}{16 \pi^{2}} \int_{\tilde{\Sigma}_{3}} \omega
$$

If $\tilde{\Sigma}_{3}$ is a circle bundle with fibre generated by an asymptotic Killing vector and if the length of the fibre at infinity is a constant over $S^{2}$ and given by $2 \pi R$, then it is convenient to define the densities

$$
\hat{P}[u]=\frac{1}{R} \tilde{P}[u], \quad \hat{K}[v]=\frac{1}{R} \tilde{K}[v]
$$

We now show that the charge $K$ indeed occurs in the superalgebra. We shall suppose that the background space $\bar{M}$ is supersymmetric, with Killing spinors $\alpha_{0}$, 
satisfying $\tilde{\nabla} \alpha_{0}=0$ (where the supervariation of the gravitino in the background $\bar{M}$ is $\left.\delta \psi_{M}=\tilde{\nabla}_{M} \epsilon\right)$; if $\bar{M}$ is flat, a frame can be chosen in which each Killing spinor $\alpha_{0}$ is constant. We shall be interested in asymptotic Killing spinors $\alpha$ on $M$, i.e. spinors on $M$ tending sufficiently fast to a Killing spinor $\alpha_{0}$ of $\bar{M}, \alpha=\alpha_{0}+O\left(1 / r^{p}\right)$ as $r \rightarrow \infty$ where $p=(D-2) / 2$. There is a supercharge $Q[\alpha]$ for each commuting Majorana asymptotic Killing spinor $\alpha$ on $M$, i.e. for each spinor on $M$ tending sufficiently fast to a Killing spinor $\alpha_{0}$ of $\bar{M}, \alpha=\alpha_{0}+O\left(1 / r^{p}\right)$ as $r \rightarrow \infty$ where $p=(D-2) / 2$. The supercharge is, to lowest order in the gravitino $\psi_{P}$,

$$
Q[\alpha]=\frac{1}{2 \Omega_{D-2}} \int_{\Sigma_{D-2}} \bar{\alpha} \Gamma^{M N P} \psi_{P} d \Sigma_{M N}
$$

For asymptotic Killing spinors to exist, it is necessary that the spin structure on $M$ agrees with that on $\bar{M}$ asymptotically. Consider a background space-time $\bar{M}$ which is the $D=5 \mathrm{KK}$ vacuum $\mathbb{R}^{3,1} \times S^{1}$. This has two spin structures, as spinors can be periodic or anti-periodic on $S^{1}$. With the periodic spin structure, the vacuum has Killing spinors, and spaces $M$ tending to $\bar{M}$ asymptotically and whose spin structure agrees with that of $\bar{M}$ asymptotically will have asymptotic Killing spinors. Then there is a positive mass theorem and a Bogomolnyi bound for such spaces, and the vacuum is stable against fluctuations which have the same spin structure asymptotically as the vacuum. On the other hand, with the antiperiodic spin structure, the vacuum has no Killing spinors and is not supersymetric, and spaces $M$ tending to $\bar{M}$ with anti-periodic spinors at infinity will have no asymptotic Killing spinors and so need not have positive mass. Indeed, there are negative mass configurations with these asymptotics, leading to the instability of the KK vacuum [61]. The 5-dimensional Euclidean Schwarzschild space tends asymptotically to the Euclidean vacuum $\mathbb{R}^{4} \times S^{1}$ with anti-periodic spin-structure, and can be continued to a Lorentzian expanding bubble solution responsible for the decay of the KK vacuum [61]. Thus stability, positivity of the mass and a BPS bound can only be established for spaces with supersymmetric boundary conditions. With non-supersymmetric asymptotics, the loss of supersymmetry 
means that there is nothing to prevent the ADM mass from becoming negative, and the vacuum is unstable. For example, the space $Y \times \mathbb{R}$, where $Y$ is $D=4$ Euclidean Schwarzschild space, does not admit asymptotic Killing spinors, since the unique spin structure on $Y \times \mathbb{R}$ requires the spinors to be anti-periodic in the $S^{1}$ at infinity generated by translations in the periodic Euclidean Schwarzschild time. On the other hand, if $Y$ is Euclidean Taub-NUT (with mass $M$ and NUT parameter $N$ ), the spinor structure is periodic and there are asymptotic Killing spinors. If $M=|N|, Y$ is self-dual and has covariantly constant spinors, while if $N=0, Y$ reduces to Euclidean Schwarzschild space and the limit $N \rightarrow 0$ leads to a change in topology and spin structure. In what follows, we shall consider only spaces with asymptotic Killing spinors, and will prove stability and a Bogomolnyi bound within this class of spaces.

For commuting asymptotic Killing spinors $\alpha, \beta$, the supercharges satisfy the algebra [33]

$$
\{Q[\alpha], Q[\beta]\}=\frac{1}{2 \Omega_{D-2}} \int_{\Sigma_{D-2}} E^{M N}(\alpha, \beta) d \Sigma_{M N}
$$

in a bosonic background (otherwise there are corrections of second order in fermion fields) where

$$
E^{M N}(\alpha, \beta)=\frac{1}{2} \bar{\alpha} \Gamma^{M N P} \hat{\nabla}_{P} \beta+\text { c.c. }
$$

where $\hat{\nabla}$ is the supercovariant derivative occurring in the gravitino supersymmetry transformation $\delta \psi_{M}=\hat{\nabla}_{M} \epsilon$ and c.c. denotes complex conjugate. In purely gravitational backgrounds, $\hat{\nabla}$ is the usual gravitational covariant derivative $\nabla$. Using the asymptotic behaviour of the spinors, (3.10) can be rewritten in terms of the charges $P, K$ as

$$
\{Q[\alpha], Q[\beta]\}=P\left[\bar{\alpha} \Gamma^{M} \beta\right]+K\left[*\left(\bar{\alpha} \Gamma^{M_{1} \ldots M_{D-5}} \beta\right)\right]+\sum_{p} \frac{1}{p !} Z_{M_{1} \ldots M_{p}} \bar{\alpha} \Gamma^{M_{1} \ldots M_{p}} \beta
$$

where the $Z_{M_{1} \ldots M_{p}}$ are the electric and magnetic anti-symmetric tensor charges, discussed in section 2 ; the values of $p$ that occur depend on the algebra, and are 
given in section 2 for $D=10,11$. If $\alpha=\beta,(3.11)$ becomes the Nestor tensor [32] which can be used to derive a BPS bound for the mass in terms of $K$ and the anti-symmetric tensor charges, as in $[15,34]^{\star}$. This then implies similar bounds for the $p$-brane densities $\tilde{P}, \tilde{K}$ and for the KK monopole densities $\hat{P}, \hat{K}$. The bound requires that the matter system satisfies a positive energy condition, and that the energy density is bounded below by the appropriate charge densities, as in $[15,34,35]$. It also requires the existence of asymptotic Killing spinors satisfying the 'Witten equation' $[15,34,35]$; when asymptotic Killing spinors exist, they can usually be chosen to satisfy the Witten equation and this has been proven in four dimensions for asymptotically flat spaces [36]. The requirement of the existence of asymptotic Killing spinors implies that the bound only applies to systems satisfying supersymmetric boundary conditions, which in particular requires that the spin structure agree asymptotically with that of a supersymmetric space.

\section{Bogomolnyi Bounds in Five Dimensions and Four Dimensions}

In the case $D=5, K$ is a scalar and so occurs as a central charge in the $D=5$ superalgebra. The $N$ extended superalgebra in $D=5$ is

$$
\begin{aligned}
\left\{Q_{\alpha}^{a}, Q_{\beta}^{b}\right\} & =\Omega^{a b}\left(\Gamma^{M} C\right)_{\alpha \beta} P_{M}+\Omega^{a b} C_{\alpha \beta} K \\
& +\left(\Gamma^{M} C\right)_{\alpha \beta} Z_{M}^{a b}+\Omega^{a b} C_{\alpha \beta} Z^{a b}+\left(\Gamma^{M N} C\right)_{\alpha \beta} Z_{M N}^{a b}
\end{aligned}
$$

Here $N$ is even, the supercharges $Q_{\alpha}^{a}$ with $\alpha=1, \ldots, 4$ and $a=1, \ldots N / 2$ are symplectic Majorana spinors and $\Omega^{a b}$ is the symplectic invariant of $U S p(N)$. The central charges $Z^{a b}$ satisfy $Z^{a b}=-Z^{b a}$ and $\Omega_{a b} Z^{a b}=0$, the charges $Z_{M}^{a b}$ satisfy $Z_{M}^{a b}=-Z_{M}^{b a}$ and $\Omega_{a b} Z_{M}^{a b}=0$ while $Z_{M N}^{a b}=Z_{M N}^{b a}$. The central charges $Z^{a b}$ arise as 0-brane charges, the $Z_{i}^{a b}$ arise as string charges, and there are also 2,3 and 4-brane charges given by $Z_{i j}^{a b}, \hat{Z}_{i j k}^{a b}$ and $\hat{Z}_{i j k l}^{a b}$, respectively. The gravitational charges are

\footnotetext{
$\star$ In certain cases, such as the IIB algebra (2.3) and the $N=2$ algebra in $D=4$, but not the $D=11$ algebra or the $I I A$ algebra, there is also a 3 -form charge arising in the gravitational sector; this will be discussed further elsewhere.
} 
the 5-momentum $P_{M}$ and the central charge $K$. For example, for $N=8$ in $D=5$, the supermultiplet contains 27 abelian gauge fields giving 27 electric charges $Z^{a b}$ and 27 magnetic string charges $Z_{i}^{a b}$. On reduction to $D=4$, the 28 electric central charges come from $P_{5}$ and $Z^{a b}$, while the 28 magnetic central charges come from $Z_{5}^{a b}$ and $K$. The $D=5, N=8$ algebra with central charge $K$ was considered in this context in [37]. The application of the positive mass theorem to $D=5 \mathrm{KK}$ monopoles was also considered in [38-41].

The algebra (4.1) gives BPS bounds for $p$-branes, in the usual way $[15,18,34,35,37]$ for spaces with supersymmetric boundary conditions with matter satisfying a 'local BPS condition' that its energy density is bounded below by an expression depending on charge densities. For 0-branes, or for asymptotically flat or KK (multi-) monopole space-times, in $D=5$, the mass satisfies

$$
M \geq\left|\lambda_{n}\right|
$$

where the $\lambda_{n}, n=1, \ldots, N / 2$ are the skew eigen-values of $Z^{a b}+K \Omega^{a b}$. In particular, this implies

$$
M \geq|K|
$$

with equality for space-times $\mathbb{R} \times N_{4}$ where $N_{4}$ is self-dual or anti-self-dual. Dividing by the size of the $S^{1}$ fibre, this gives

$$
\hat{M} \geq|\hat{K}|
$$

and as we shall see in section $5, \hat{K}$ can be identified with the NUT charge $N$. Dimensionally reducing with respect to the time coordinate to $N_{4}$ gives the bound

$$
\hat{M} \geq|N|
$$

for four-dimensional Euclidean geometries with asymptotic Killing spinors with equality for spaces admitting covariantly constant spinors, i.e. for self-dual geometries. This can be obtained directly by considering the dimensional reduction of 
(3.10) to $N_{4}$ (i.e. reducing in the time direction) given by

$$
\frac{1}{2 \Omega_{3}} \int_{\tilde{\Sigma}_{3}} E^{M N}(\alpha, \beta) d \Sigma_{M N}
$$

where

$$
E^{M N}(\alpha, \beta)=\frac{1}{2} \bar{\alpha} \Gamma^{5} \Gamma^{N P} \hat{\nabla}_{P} \beta+c . c .
$$

The bound then follows from standard arguments using $E^{M N}(\alpha, \alpha)$. For ALF boundary conditions, $\tilde{\Sigma}_{3}$ is a circle bundle over $S^{2}$. The quantity $\hat{M}$ is the ADM momentum $P[k]$ corresponding to the asymptotic Killing vector $k$ generating the $S^{1}$ fibre. Including electric and magnetic charges $Q, P$, this becomes

$$
\hat{M}^{2}+Q^{2} \geq N^{2}+P^{2}
$$

This can formally be continued to Lorentzian signature metrics. Wick rotating the $S^{1}$ fibres (i.e. in adapted coordinates in which $k=\partial / \partial y$, we Wick rotate the coordinate $y \rightarrow i t)$ an argument similar to that of [15] gives

$$
\hat{M}^{2}+N^{2} \geq Q^{2}+P^{2}
$$

with equality only for spaces admitting supercovariantly constant spinors $\alpha, \hat{\nabla} \alpha=$ 0 . Such a bound was suggested in [42]. 


\section{Evaluation of $K[v]$ and Relation to NUT Charge.}

Consider a $D$ dimensional manifold $M$ with an isometry generated by a space-like Killing vector $k=\partial / \partial y$. The main examples considered here will be $M=N_{4} \times \mathbb{R}^{D-5,1}$ where $N_{4}$ is 4-dimensional Taub-NUT space or its multi-centre generalisation. The isometry defines a fibering $\pi: M \backslash \mathcal{F} \rightarrow B$ where $\mathcal{F}$ is the fixed-point set of the action of $G$, so that $B$ is the space of non-degenerate orbits. The metric $G_{M N}$ on $M$ induces a Lorentzian signature metric

$$
g_{M N}=G_{M N}-V^{-1} k_{M} k_{N}
$$

on $B$, where $V=k^{M} k_{M}$. The metric $G_{M N}$ on $M$ can be written as

$$
d s^{2}=V\left(d y+A_{\mu} d x^{\mu}\right)^{2}+V^{-1} g_{\mu \nu} d x^{\mu} d x^{\nu}
$$

where $V, A_{\mu}$ and $g_{\mu \nu}$ do not depend on the coordinate $y$. The vector field $A_{\mu}$ is defined up to a gauge transformation

$$
A_{\mu} \rightarrow A_{\mu}+\partial_{\mu} \rho
$$

as such a change can be absorbed into the coordinate transformation

$$
y \rightarrow y-\rho
$$

The invariant field-strength

$$
F_{\mu \nu}=\partial_{[\mu} A_{\nu]}
$$

is the twist or vorticity of the vector field $k$ and can be written covariantly in $D$ dimensions as

$$
F_{M N}=V^{-1} g_{M}^{P} g_{N}^{Q} \nabla_{P} k_{Q}
$$

On dimensional reduction with respect to the Killing vector $k$, the $D$ dimensional metric $g_{M N}$ gives a metric $g_{\mu \nu}$, a graviphoton $A_{\mu}$ and a graviscalar 
$V$ on the $D-1$ dimensional space $B$. The kinetic term for the metric $g_{\mu \nu}$ is not conventionally normalised; the Einstein metric is given by $g_{\mu \nu}^{E}=V^{\alpha} g_{\mu \nu}$ where $\alpha=-1 / D-2$. Note that the two metrics $g_{\mu \nu}$ and $g_{\mu \nu}^{E}$ lead to different ADM momenta, $P_{\mu}$ and $P_{\mu}^{E}$ respectively [40]. The vector field $A_{\mu}$ is an abelian gauge field on $B$, which will have electric charge $q$ and a magnetic charge given by a $D-5$ form $Z_{m_{1} \ldots m_{D-5}}$, where $x^{m}$ are the spatial coordinates on $B$, with $m=1, \ldots, D-2$. If the boundary of a spatial slice of $B$ is $\mathbb{R}^{D-5} \times \Sigma^{2}$ or $T^{D-5} \times \Sigma^{2}$ for some compact 2 -space $\Sigma^{2}$, then

$$
Z . v=\frac{1}{4 \pi} \int_{\Sigma^{2}} F
$$

where $v$ is the volume-form on $\mathbb{R}^{D-5}$. If $M=N_{4} \times \mathbb{R}^{D-5,1}$, then the NUT charge defined in $[30,31,45]$ can be generalised to a $D-5$ form charge $N_{\mu_{1} \ldots \mu_{D_{5}}}[k]$ for $M=N_{4} \times \mathbb{R}^{D-5,1}$ (or for the Euclidean space $M=N_{4} \times \mathbb{R}^{D-5}$ ) given by

$$
N[k] \cdot v=\frac{1}{8 \pi} \int_{\Sigma^{2}} F=\frac{1}{2} Z \cdot v
$$

(The factor of $8 \pi$ in (5.8) is included to agree with the normalisation in [30,31,45].) If $y$ is periodic with period $P, y \sim y+P$, then the length of the orbit of $k$ at $x^{\mu}$ is $\sqrt{V(x)} P$ which tends to $2 \pi R$, say, on $\Sigma$. The electric charge $q$ of the graviphoton is proportional to the momentum in the $y$ direction $q \propto \frac{1}{R} P^{E}[k]$, while, as we shall see, the magnetic charge is $Z . n \propto \frac{1}{2 R} K[n]$.

The momentum $P[k]$ and the corresponding NUT charge satisfy a Dirac quantization condition and the relation of the NUT charge to mass is similar to the relation of magnetic charge to electric charge. Two generalisations of the NUT charge or dual mass to spaces without isometries are given in [30,31]. However, both invoke special structures at infinity, whereas the definition of the charge $K[v]$ is general and needs no such structures. 
Let $e_{M}^{A}$ and $\hat{e}_{\mu}^{a}$ be frames on $M, B$ respectively, satisfying

$$
e_{M}^{A} e_{N}^{B} \eta_{A B}=G_{M N}, \quad \hat{e}_{\mu}^{a} \hat{e}_{\nu}^{b} \eta_{a b}=V^{-1} g_{\mu \nu}
$$

A suitable choice of one-forms $e^{A}=\left\{e^{y}, e^{a}\right\}$ is

$$
e^{y}=V^{1 / 2}\left(d y+A_{\mu} d x^{\mu}\right), \quad e^{a}=\hat{e}_{\mu}^{a} d x^{\mu}
$$

The corresponding spin-connection one-forms $\omega^{A}{ }_{B}, \hat{\omega}^{a}{ }_{b}$ satisfy

$$
d e^{A}+\omega^{A}{ }_{B \wedge} e^{B}=0, \quad d \hat{e}^{a}+\hat{\omega}^{a}{ }_{b \wedge} \hat{e}^{b}=0
$$

so that

$$
\begin{aligned}
\omega^{y}{ }_{a} & =\frac{1}{2} V^{-1} V_{a} e^{y}+V^{1 / 2} F_{a b} e^{b}, \\
\omega^{a}{ }_{b} & =\hat{\omega}^{a}{ }_{b}-V^{1 / 2} F_{a b} e^{y}
\end{aligned}
$$

where $V_{a}=\partial_{\mu} V e_{a}^{\mu}$ and $F_{a b}=e_{a}^{\mu} e_{b}^{\nu} F_{\mu \nu}$ with $F_{\mu \nu}=2 \partial_{[\mu} A_{\nu]}$. Then the 3 -form corresponding to the totally antisymmetric part of the spin-connection is

$$
\omega=F_{\wedge} k+\hat{\omega}
$$

where

$$
k=V\left(d y+A_{\mu} d x^{\mu}\right)=k_{M} d x^{M}, \quad \hat{\omega}=e_{\wedge}^{b} e^{a} \hat{\omega}_{a b}
$$

and $F=\frac{1}{2} F_{\mu \nu} d x_{\wedge}^{\mu} d x^{\nu}$. In what follows, we shall consider only the case in which the contribution $\hat{\omega}$ to (5.13) from the geometry of $B$ is zero. Such contributions could have arisen, for example, if there were several Killing vectors giving rise to several magnetic charges, and their treatment is a straightforward generalisation of the case considered here. (Alternatively, instead of considering asymptotics with respect to a flat background, we could consider asymptotics with respect to a background with $\bar{\omega}_{b}^{a}=\hat{\omega}^{a}{ }_{b}$, so that $\omega \equiv e_{\wedge}^{b} e_{\wedge}^{a}\left(\omega_{a b}-\bar{\omega}_{a b}\right)=F_{\wedge} k$. $)$ 
The isometry defines a fibering $\pi: \tilde{\Sigma}_{3} \backslash \mathcal{F}^{\prime} \rightarrow \Sigma^{2}$ where $\Sigma_{D-2}=T^{D-5} \times \tilde{\Sigma}_{3}$ or $\Sigma_{D-2}=\mathbb{R}^{D-5} \times \tilde{\Sigma}_{3}$ is the boundary of a spatial slice of $M$ and $T^{D-5} \times \Sigma^{2}$ or $\mathbb{R}^{D-5} \times \Sigma^{2}$ is the boundary of a spatial slice of $B$, and $\mathcal{F}^{\prime}$ is the fixed-point set of the isometry action. Then $F_{\wedge} k$ is proportional to the volume form of $\tilde{\Sigma}_{3}$, so that $\int \omega$ is a winding number. From (5.13) and the fact that $k$ is a Killing vector, it follows that

$$
\tilde{K}=\frac{1}{16 \pi^{2}} \int_{\tilde{\Sigma}_{3}} \omega=\frac{1}{16 \pi^{2}} \int_{S^{1}} k \int_{\tilde{\Sigma}} F=\frac{R}{8 \pi} \int_{\Sigma} F=R N
$$

where $2 \pi R=\int k$ is the length of the fibre at infinity. In particular, this implies that

$$
\hat{K}=N
$$

In the case of Taub-NUT, $\tilde{\Sigma}_{3}$ is $S^{3}$ and $\Sigma^{2}=S^{2}$, with $\pi$ the Hopf fibration. This case will now be examined in detail.

The Euclidean Taub-NUT metric is of the form (5.2); taking $x^{\mu}$ as spherical polar coordinates $r, \theta, \phi$, it can be written as

$$
d s^{2}=V\left(d y+A_{\phi} d \phi\right)^{2}+V^{-1} d r^{2}+r^{2} f\left(d \theta^{2}+\sin ^{2} \theta d \phi^{2}\right)
$$

where

$$
V=1-\frac{2\left(M r+N^{2}\right)}{r^{2}+N^{2}}, \quad A_{\phi}=4 N \sin ^{2} \frac{\theta}{2}, \quad f=1-\frac{2 M}{r}+\frac{N^{2}}{r^{2}}
$$

The Killing vector $k=\partial / \partial y$ can be used to define a component of momentum $P[k]$ and a NUT charge $N[k]$ given by the parameters $M, N$ respectively. The limit $N \rightarrow 0$ gives Euclidean Schwarzschild with 'Euclidean mass' $M$ (i.e. the mass resulting from regarding $y$ as the Euclidean time). The parameter $M$ is the $k$-component of momentum $P[k]$ and $N$ is the corresponding NUT charge. The curvature is self-dual if $M=N$ and anti-self-dual if $M=-N$. Regarded as a 
metric on $\mathbb{R}^{4}$ with spherical polar coordinates $\theta, \phi$, this metric has a Dirac string or wire singularity along the half-axis $\theta=\pi$. This singularity can be removed by introducing a new coordinate

$$
y^{\prime}=y+4 N \phi
$$

The metric becomes

$$
d s^{2}=V\left(d y^{\prime}+A_{\phi}^{\prime} d \phi\right)^{2}+V^{-1} d r^{2}+r^{2} f\left(d \theta^{2}+\sin ^{2} \theta d \phi^{2}\right)
$$

with

$$
A_{\phi}^{\prime}=-4 N \cos ^{2} \frac{\theta}{2}
$$

The field strength $F=d A=d A^{\prime}$ is well-defined and given by

$$
F=2 N \sin \theta d \theta_{\wedge} d \phi
$$

The metric (5.20) is regular at $\theta=\pi$ but not at $\theta=0$. A non-singular metric is obtained by using $t, r, \theta, \phi$ in the patch $0 \leq \theta<\pi$ and $t^{\prime}, r, \theta, \phi$ in the patch $0<$ $\theta \leq \pi$. In the overlap, the transition (5.19) is consistent with the periodicity of $\phi$ if we make the $y$ coordinate periodic with period $8 \pi|N|$. This changes the topology of the surfaces $r=$ constant from $S^{2} \times \mathbb{R}$ to $S^{3}$ and leads to the requirement that the energy $E$ of any particle moving in the space-time has to satisfy the Dirac quantization condition $4 N E=$ integer. Then $\psi, \phi, \theta$ are Euler angles on $S^{3}$ with $\psi=y / 2|N|$. The $S^{3}$ has a squashed metric: as $r \rightarrow \infty$, the length of the $S^{1}$ Hopf fibres tends to a constant, $8 \pi|N|$, while the area of the $S^{2}$ base increases as $r^{2}$.

The three-form $\omega$ is given by

$$
\omega=2 N V \sin \theta d \theta_{\wedge} d \phi_{\wedge} d y=2 N V \sin \theta d \theta_{\wedge} d \phi_{\wedge} d y^{\prime}=4 N|N| V \sin \theta d \theta_{\wedge} d \phi_{\wedge} d \psi
$$

and is proportional to the volume form on $S^{3}$ (and is well-defined). Integrating on 
an $S^{3}$ of constant $r$ and taking the limit $r \rightarrow \infty$ gives

$$
\tilde{K}=\frac{1}{16 \pi^{2}} \int \omega=4 N|N|
$$

The $S^{3}$ is a circle bundle over the 2 -sphere $\Sigma$ parameterised by $\theta, \phi$ with fibre coordinate $\psi$. The NUT charge $(5.8)$ is

$$
\frac{1}{8 \pi} \int_{\Sigma} F=\frac{1}{8 \pi} \int_{\Sigma} 2 N \sin \theta d \theta d \phi=N
$$

so that they are indeed related by $K=R N, \hat{K}=N$, where $R$ is the radius of the $S^{1}, R=4|N|$.

The self-dual multi-Taub-NUT solution [28] is also of the form (5.2), but with

$$
V^{-1}=1+\sum_{i=1}^{s} \frac{2 N_{i}}{\left|x^{\mu}-x_{i}^{\mu}\right|}, \quad F_{\mu \nu}=\epsilon_{\mu \nu \rho} \nabla^{\rho} V^{-1}
$$

and with

$$
g_{\mu \nu}=\delta_{\mu \nu}
$$

and this flat metric is used to raise and lower three-dimensional indices $\mu, \nu, \ldots$.. There are $s$ nuts with parameters $N_{i}$ at the positions $x_{i}^{\mu}$. The potential $A$ will again have Dirac string singularities in general, but if all the parameters $n_{i}$ are equal to a single value $N$, then all singularities are removed by identifying $y$ with period $8 \pi|N|$. The surface at infinity is the Lens space $L(s, 1)$. As $\left|x^{\mu}\right| \rightarrow \infty$,

$$
V \rightarrow 1, \quad F \rightarrow 2 N s \sin \theta d \theta_{\wedge} d \phi, \quad \omega \rightarrow 2 N s \sin \theta d \theta_{\wedge} d \phi_{\wedge} d y
$$

so that integrating $\omega$ over the Lens space $L(s, 1)$ at infinity gives

$$
\tilde{K}=\frac{1}{16 \pi^{2}} \int \omega=\frac{1}{s} \frac{1}{16 \pi^{2}} \int 2 N s \sin \theta d \theta d \phi d y=4 N|N|
$$


while the NUT charge is

$$
N=\frac{1}{8 \pi} \int_{\Sigma} F=\frac{1}{8 \pi} \int_{\Sigma} 2 N s \sin \theta d \theta d \phi=s N
$$

so that they are related by $K=R N$, where $R$ is the 'size' of the fibre $S^{1} / \mathbb{Z}_{s}$, $R=4|N| / s$.

\section{Nine-Branes in IIB Theory}

We now turn to the new 9-branes that have been proposed for the type IIB theory. That such branes should be present in the theory follows from duality and supersymmetry. It is known that there are D-9-branes with RR charge in the type IIB string, and that they break half the supersymmetry, so that there should be a corresponding 9-brane charge in the IIB superalgebra. Consider now the action of an $S L(2, \mathbb{Z})$ duality transformation on the RR 9-brane. The 9-brane will either be invariant, or will be mapped into a new 9-brane. However, we have seen from the superalgebra that the 9-brane charges that occur on the right hand side of (2.3) fit into doublets of $S O(2)$, as do the 1-brane and 5-brane charges. For the 1brane and 5-brane, the $S O(2)$ doublet of charges is related (by a scalar-dependent transformation) to an $S L(2)$ doublet of charges, and in the same way the $S O(2)$ doublet of 9-brane charges $\left(Z^{1}, Z^{2}\right)$ can be converted to an $S L(2)$ doublet of 9brane charges $\left(\bar{Z}^{1}, \bar{Z}^{2}\right)$. The D-9-brane charge $\bar{Z}^{2}$ is then not a singlet of $S L(2, \mathbb{Z})$, so the D-9-brane is mapped to a new 9-brane, and the action of $S L(2, \mathbb{Z})$ on the doublet of 9-brane charges will give $(p, q)$ 9-branes with $p, q$ co-prime integers.

In the type IIB theory, the RR 9-brane occurs as a D-brane and gives rise to Chan-Paton factors for the fundamental string at weak coupling. A fundamental string can end on a D-brane, and the standard configuration with $n$ 9-D-branes would be with the $n$ branes coincident and each filling $D=10$ space-time. Thus strings ending on a 9-brane would be open strings, with each end labelled by which of the $n$ 9-D-branes it ended on, giving rise to an $n$-dimensional Chan-Paton factor. 
In $D=10$ Minkowski space, charge conservation forbids the presence of any 9-Dbranes. However, if one mods out by world-sheet parity reversal $\Omega$ to construct an orientifold, the whole $D=10$ Minkowski space is a source of 9-brane charge which can be cancelled by adding precisely 32 9-D-branes, giving rise to the type I string with $S O(32)$ Chan-Paton factors.

As the presence of 9-branes is usually not allowed without the presence of some other sources of charge, such as an orientifold, any discussion of 9-branes in general is necessarily rather formal. Before discussing the possibility of generalising the usual orientifolds to give sources of $(p, q)$ 9-brane charge so as to give consistent backgrounds with $(p, q)$ 9-branes, it will be useful to consider 9-branes and their relations to other branes further.

The type IIB theory has an $S L(2, \mathbb{Z})$ symmetry $[2,10]$ which acts on the complex scalar $\lambda=\chi+i e^{-\Phi}$, where $\Phi$ is the dilaton and $\chi$ is the RR scalar, and the two anti-symmetric tensor field strengths $H_{M N P}^{u}$ with $u=1,2$ where $H^{1}=d B^{1}$ is the NS-NS field strength and $H^{2}=d B^{2}$ is the RR field strength. The transformations are

$$
\lambda \rightarrow \frac{p \lambda+q}{r \lambda+s}, \quad\left(\begin{array}{c}
H^{1} \\
H^{2}
\end{array}\right) \rightarrow \Lambda\left(\begin{array}{l}
H^{1} \\
H^{2}
\end{array}\right)
$$

where $\Lambda$ is the $S L(2, \mathbb{Z})$ matrix

$$
\Lambda=\left(\begin{array}{ll}
p & r \\
q & s
\end{array}\right), \quad p s-q r=1, \quad p, q, r, s \in \mathbb{Z}
$$

The branes of the type IIB theory fit into representations of the $S L(2, \mathbb{Z})$ symmetry. Some are singlets, while some of the branes transform as doublets of the $S L(2, \mathbb{R})$ symmetry of the classical supergravity theory leading in the quantum theory to a spectrum of branes with charge $(p, q)$ with $p, q$ co-prime integers. The 3-brane is a singlet and the strings and 5-branes are doublets. The 7-brane is more subtle, as it couples to the scalar fields which transform non-linearly under $S L(2, \mathbb{Z})$. The solutions of [23] in which the axion ansatz involves the modular invariant $j$ function are $S L(2, \mathbb{Z})$ invariant and lead to singlet 7-brane charges. Acting with 
$S L(2, \mathbb{Z})$ on a 7 -brane leaves its charge $Z_{i_{1} \ldots i_{7}}$ invariant, but changes the $S L(2, \mathbb{Z})$ monodromy and the couplings to strings and 5-branes [26]. This is consistent with the superalgebra, which we have seen has singlet 3-brane and 7-brane charges, and 1-brane and 5-brane charges that are doublets. The 9-brane charges are doublets, so that there should be 9-branes with charges $(p, q)$ with $p, q$ co-prime.

Strings carry two one-form charges $\bar{Z}_{i}^{u}$ and 5-branes carry two 5-form charges $\bar{Z}_{i j k l m}^{u}$, which transform as doublets under $S L(2)$;

$$
\bar{Z}_{i}=\left(\begin{array}{c}
\bar{Z}_{i}^{1} \\
\bar{Z}_{i}^{2}
\end{array}\right), \quad \bar{Z}_{i j k l m}=\left(\begin{array}{c}
\bar{Z}_{i j k l m}^{1} \\
\bar{Z}_{i j k l m}^{2}
\end{array}\right)
$$

The scalars can be used to construct a $2 \times 2$ matrix $\mathcal{V} \in S L(2, \mathbb{R})$ transforming under rigid $S L(2, \mathbb{R})$ from the right and under local $S O(2)$ from the left, so that under $S L(2, \mathbb{Z}), \mathcal{V} \rightarrow \mathcal{V} \Lambda^{-1}$. Fixing an $S O(2)$ gauge gives an expression for If $\mathcal{V} \rightarrow \mathcal{V}_{0}$ at infinity, for $\mathcal{V}$ in terms of the complex scalar $\lambda$. some constant matrix $\mathcal{V}_{0}$, then $Z_{i}=\mathcal{V}_{0} \bar{Z}_{i}$ and $Z_{i j k l m}=\mathcal{V}_{0} \bar{Z}_{i j k l m}$ are $S L(2)$-invariant charges which however transform as doublets under the $S O(2)$ which also acts on the fermions and supercharges; it is these charges $Z_{i}, Z_{i j k l m}$ that enter into the superalgebra (2.3).

A similar structure should hold for the 9-branes. The 2-vector of 9-brane charges $Z_{0}$ (dual to $\hat{Z}_{i_{1} \ldots i_{9}}^{a b}$ ) in the algebra is an $S O(2)$ doublet and should arise from an $S L(2)$ doublet $\bar{Z}_{0}$, with $Z_{0}=\mathcal{V}_{0} \bar{Z}_{0}$. The D-brane with $p=9$ can be thought of as coupling to a 10 -form potential $A^{2}$ which is an auxiliary field of the theory, occurring in the RR sector. (Strictly speaking, the RR construction gives only physical fields, but the 9-brane coupled to the 10-form is related by T-duality to $p$-branes coupling to $\mathrm{RR}(p+1)$ form gauge fields.) As the charges $\bar{Z}_{0}$ are an $S L(2)$ doublet, there should be an $S L(2)$ doublet of 10-form potentials, $A^{1}, A^{2}$. The field $A^{1}$ is again non-physical, and it seems natural to attribute it to some generalisation of the usual NS-NS sector to include auxiliary fields.

At weak coupling, $g \equiv<e^{\Phi}>\approx 0$, the perturbative states are described by the NS-NS or $(1,0)$ string while all the other branes are non-perturbative [7]. The 
perturbative theory is formulated as the usual type IIB superstring theory, with a topological expansion in terms of the genus of the world-sheet of the NS-NS string. At strong coupling, however, it is the RR or $(0,1)$ string that gives the states that are perturbative in an expansion in $\tilde{g}=1 / g$. The conjectured self-duality of the theory implies that the strong-coupling theory is again a type IIB string theory, but now the formulation should be in terms of the world-sheet of the $(0,1)$ string. The perturbation theory in $\tilde{g}$ is a sum over $(0,1)$ string world-sheets with the power of $\tilde{g}$ corresponding to the genus of the world-sheet. At weak coupling, the $(1,0)$ string can end on the D-branes carrying RR charge, which are the 3-brane, the 7-brane, the $(0,1)$ string, the $(0,1) 5$-brane and the RR 9-brane, which we will refer to as the $(0,1)$ 9-brane. In the strongly coupled theory formulated in terms of the world-sheet of the $(0,1)$ string, the $(0,1)$ string and $(0,1) 5$-brane carry charge that appears in the NS-NS sector of the dual $(0,1)$ string (as this couples to $B^{2}$ ) while the new D-branes with density proportional to $1 / \tilde{g}$ on which the $(0,1)$ string can end are the 3 -brane, the 7 -brane, the $(1,0)$ string and the $(1,0) 5$-brane. These all carry charge which occurs in the RR sector of the $(0,1)$ string. This structure is obtained by acting with the $S L(2, \mathbb{Z})$ transformation generated by

$$
S=\left(\begin{array}{cc}
0 & 1 \\
-1 & 0
\end{array}\right)
$$

which interchanges strong and weak coupling. For example, this takes a $(1,0)$ string ending on $(0,1)$ strings or 5 -branes to a $(0,1)$ string ending on $(1,0)$ strings or 5-branes.

This should extend to 9-branes as follows. There are two basic 9-branes, with charges $(1,0)$ and $(0,1)$. At weak coupling, the $(0,1)$ 9-brane is a D-brane for the weakly coupled $(1,0)$ string and carries RR charge. The strongly coupled theory is formulated in terms of the world-sheet of the $(0,1)$ string and has the $(1,0)$ 9-brane as a D-brane occurring in the RR sector of the $(0,1)$ string. A general 9-brane has

\footnotetext{
$\star$ The $(1,0)$ string can also end on $(n, \pm 1)$ strings, 5-branes and 9-branes, as follows from $S L(2, \mathbb{Z})$ duality. However, it will be sufficient here to focus on the $(0,1)$ Dirichlet branes.
} 
two charges $(p, q)$ corresponding to the two 10-forms $A^{1}, A^{2}$ and can be thought of as a bound state of $(1,0)$ and $(0,1)$ branes. The two auxiliary 10 -form fields fit into an $S L(2, \mathbb{Z})$ doublet and one occurs in the RR sector of the $(1,0)$ string, while the other occurs in the RR sector of the $(0,1)$ string. (A naive extrapolation of the formulae of [7] suggests that for the weakly coupled string, the coupling constant dependence of the density of the NS-NS 9-brane is $g^{-4}$. This is to be compared with that of the RR 9-brane and other D-branes which have densities of order $g^{-1}$ and that of the solitonic NS-NS 5-brane which has density of order $g^{-2}$. Then on going to strong coupling and using the dual string metric, the (1,0) 9-brane would have density $\tilde{g}^{-1}$ and the $(0,1)$ 9-brane would have density $\tilde{g}^{-4}$. However, this is rather formal as there are various problems in discussing the densities of $p$-branes with $p \geq 7$ in ten dimensions.)

Instead of acting with the $S L(2, \mathbb{Z})$ transformation (6.4) that takes us from weak to strong coupling, we could act with a general $S L(2, \mathbb{Z})$ transformation generated by (6.2). The $(1,0) 1,5,9$-branes are mapped to $(p, q) 1,5,9$-branes and the $(0,1) 1,5,9$-branes are mapped to $(r, s)$ branes, while the transformation (6.1) of $\lambda$ leads to a new coupling constant $\tilde{g}$. Perturbation theory in $\tilde{g}$ again gives a type IIB string theory, but where now the $(p, q)$ string is fundamental and formulated in terms of the $(p, q)$ string world-sheet. The D-branes of this dual world-sheet description are the $(r, s)$ 1-branes, 5-branes and 9-branes, together with the 3brane and the 7-brane. These couple to potentials that arise in the RR sector of the new fundamental string. The $(p, q) 5$-brane is now solitonic, and other strings and 5-branes occur as various bound states.

We turn now to the orientifold construction. At weak coupling, the type IIB theory is formulated in terms of a fundamental $(1,0)$ string and has a symmetry $\Omega$ which reverses the parity of the $(1,0)$ world-sheet. As the dilaton is invariant under $\Omega$, this is a perturbative symmetry. The massless bosonic fields of the IIB theory are $g_{M N}, B_{M N}^{1}, \Phi$ in the NS-NS sector and $D_{M N P Q}, B_{M N}^{2}, \chi$ in the RR sector. Of these, the ones that are invariant under $\Omega$ are $g_{M N}, B_{M N}^{2}, \Phi$. The orientifold of the IIB theory constructed using $\Omega$ gives the type I theory [12]. 
The invariant sector is formulated in terms of closed type I strings, with massless bosonic fields $g_{M N}, B_{M N}^{2}, \Phi$. In addition there is an open string sector, which can in some ways be thought of as a twisted sector, with $S O(32)$ Chan-Paton factors arising from 32 RR 9-branes [13]. Acting with $\Omega$ projects out all branes except the RR string, 5-brane and 9-brane, although there is a sense in which the closed and open fundamental type I strings can be associated with the fundamental NS-NS string of the type IIB theory.

Consider now the strongly coupled type IIB string, formulated as a world-sheet theory of the $(0,1)$ string. This should be isomorphic to the usual type IIB string, and in particular has a symmetry $\tilde{\Omega}$ which reverses the parity of the $(0,1)$ string world-sheet. As $\tilde{\Omega}$ leaves the dilaton invariant, this is a perturbative symmetry in $\tilde{g}$ perturbation theory. We can consider orientifolding by $\tilde{\Omega}$, which should make sense in $\tilde{g}$ perturbation expansion. It should be isomorphic to the usual orientifold construction, and lead to a theory isomorphic to the usual type-I theory, and in particular should have open strings and $S O(32)$ gauge symmetry. The massless bosonic fields that are invariant under $\tilde{\Omega}$ are $g_{M N}, B_{M N}^{1}, \Phi$, which occurred in the NS-NS sector of the $(1,0)$ string for $g<<1$. The Chan-Paton factors are now carried by the 9-branes that are the D-branes for the $(0,1)$ string; these are precisely the $(1,0)$ 9-branes that we have postulated.

The next question is that of how the 'new' type I string is related to the usual one. It is isomorphic to the usual one, but it fits differently into the type IIB theory - one is obtained by the usual orientifold construction, the other by doing an $S L(2, \mathbb{Z})$ transformation and then orientifolding. The two type I theories are physically indistinguishable; both are theories of non-orientible closed strings coupled to open strings with $S O(32)$ Chan-Paton factors and both have $N=1$ supergravity coupled to $S O(32)$ super-Yang-Mills as the low energy effective field theory. On modding out by $\Omega$, the $(0,1)$ string, 5 -brane and 9-brane of the IIB theory survive as type I D-branes, while the $(1,0)$ string which is fundamental for $g<<1$ leads to the fundamental type I strings which can end on the D-branes. The strong coupling limit of this type I theory is conjectured to be the $S O(32)$ 
heterotic theory [4], with the $(0,1)$ string becoming the fundamental heterotic string at strong coupling $[10,11,13]$. On the other hand, on modding out by $\tilde{\Omega}$ for $g>>1$, the $(1,0)$ string, 5 -brane and 9-brane of the IIB theory survive as the new type I D-branes, while the $(0,1)$ string which is fundamental in the IIB theory for $\tilde{g}<<1$ leads to the fundamental strings of this new type I theory which can end on the (1,0) string, 5-brane and 9-brane.

To proceed further, we shall suppose that the perturbative parity reversal symmetry $\Omega$ extends to a $\mathbb{Z}_{2}$ symmetry of the full non-perturbative type IIB theory, which we will again denote as $\Omega$. We know how this acts on perturbative states and hence on the effective low-energy supergravity theory $-g_{M N}, B_{M N}^{2}, \Phi$ are even and $D_{M N P Q}, B_{M N}^{1}, \chi$ are odd under $\Omega$ - and this tells us how it acts on BPS states: the $(0,1)$ string, 5 -brane and 9-brane are invariant while the the $(1,0)$ string, 5 -brane and 9-brane together with the 7-brane and 3-brane are odd under $\Omega$. If there is such a non-perturbative symmetry $\Omega$ it should in particular be a symmetry for all values of the coupling $g$. Then $\tilde{\Omega}$ should also extend to a non-perturbative symmetry and should be related to $\Omega$ by

$$
\tilde{\Omega}=S \Omega S^{-1}
$$

where $S$ is the $S L(2, \mathbb{Z})$ transformation (6.4). Modding the non-perturbative IIB theory by the $\mathbb{Z}_{2}$ symmetry $\Omega$ (or $\tilde{\Omega}$ ) should then make sense for any value of the coupling $g$ and would give a theory with $N=1$ supersymmetry in $D=10$ and $S O(32)$ gauge symmetry. Modding out by $\Omega$ requires a background with 32 $(0,1)$ 9-branes (since it does for weak coupling, it must for strong coupling also) while modding out by $\tilde{\Omega}$ requires $32(1,0)$ 9-branes. As modding out by $\Omega$ at weak coupling $(g<<1)$ gives the type I string, and the strong coupling limit of this is the $S O(32)$ heterotic string, this implies that modding the strongly coupled IIB string (with $32(0,1)$ 9-branes) out by $\Omega$ should give the heterotic string with coupling constant $\tilde{g}=1 / g$. Similarly, modding out the weakly coupled type IIB string (with $32(1,0)$ 9-branes) by $\tilde{\Omega}$ should give the weakly coupled heterotic string 
with coupling constant $g$. The NS-NS sector of the type IIB string $g_{M N}, B_{M N}^{1}, \Phi$ gives the $N=1$ supergravity fields of the heterotic string and in particular the type IIB dilaton is identified with the heterotic dilaton.

The symmetry $\tilde{\Omega}$ should be a perturbative symmetry of the weakly coupled IIB string. The weakly coupled type IIB string also has a perturbative symmetry $(-1)^{F_{L}}$ where $F_{L}$ is the left-handed fermion number of the conventional NSR formulation of the type IIB theory. It also leaves $g_{M N}, B_{M N}^{1}, \Phi$ invariant - the same sector preserved by $\tilde{\Omega}$. This implies that $(-1)^{F_{L}}$ and $\tilde{\Omega}$ act in the same way on BPS states - D-branes are odd and (1,0) strings and 5-branes are even under both. This suggests that $(-1)^{F_{L}}$ is the same symmetry as $\tilde{\Omega}$, and this could be taken as the definition of the extension of $(-1)^{F_{L}}$ to a symmetry of the full non-perturbative theory. Indeed, in [46] it was argued that $(-1)^{F_{L}}=S \Omega S^{-1}$ by considering the action on massless fields, while here $\tilde{\Omega}=S \Omega S^{-1}$ by definition. Similarly, extrapolating $\Omega$ to strong coupling should give $(-1)^{F_{L}^{\prime}}$ where $F_{L}^{\prime}$ is the left-handed fermion number of the NSR formulation of the dual type IIB theory, acting on fermions moving on the $(0,1)$ string world-sheet.

It has been suggested (see e.g. [46]) that modding out the type IIB theory by $(-1)^{F_{L}}$ should give the type IIA theory by considering the action on perturbative states, while we have argued that modding out by $\tilde{\Omega}$ should give a heterotic/type I theory. If $(-1)^{F_{L}}$ and $\tilde{\Omega}$ are in fact the same symmetry and if it is indeed the case that modding the type IIB theory by $(-1)^{F_{L}}$ can give the type IIA theory, then this would mean that there are two different ways of modding the IIB theory by the same symmetry, in which different 'twisted sectors' are added to obtain a consistent theory. In both cases the untwisted or invariant sector includes the $g$-perturbative states from the product of the left-handed NS sector with the right-handed R and NS sectors. This theory is inconsistent as it stands, but a consistent theory can be obtained by adding a 'twisted sector'. One way of doing this is by introducing a left-handed $\mathrm{R}$ sector of the opposite chirality, to obtain a type IIA theory, while another is to introduce $32(1,0)$ 9-branes and a heterotic sector which becomes the open string sector of a type I theory as $g \rightarrow \infty$. If this is correct, it would also 
mean that on taking an orientifold of the type IIB theory with $\Omega$, the resulting theory can be fixed up either in the usual way to obtain the type I string, or by adding a IIA twisted sector so that the theory becomes the weakly coupled IIA theory as the IIB coupling becomes large, so that the IIA and IIB couplings are inversely related. As the IIA theory becomes M-theory at strong coupling [4], this would mean that the twisted sector added to the orientifolded type IIB theory at weak coupling should be 11-dimensional. (It is of course conceivable that $(-1)^{F_{L}}$ and $\tilde{\Omega}$ are in fact two different $\mathbb{Z}_{2}$ symmetries, which have the same action on the massless sector, but differ in their actions on the full theory; modding out by one gives the type IIA theory and modding out by the other gives a type I theory. This would mean that $X=(-1)^{F_{L}} . \tilde{\Omega}$ should be some as yet unknown symmetry that preserves the massless and BPS sectors but acts non-trivially on the full theory, so that $\tilde{\Omega}=(-1)^{F_{L}} . X$.)

This can be generalised to consider orientifolding the $(p, q)$ string instead of the $(1,0)$ or $(0,1)$ string. Acting with an $S L(2, \mathbb{Z})$ transformation $\Lambda$ gives the discrete symmetry

$$
\Omega_{\Lambda}=\Lambda \Omega \Lambda^{-1}
$$

If $\Lambda$ takes a $(1,0)$ string to a $(p, q)$ string and takes the coupling constant $g$ to $g_{\Lambda}$, then perturbation theory in $g_{\Lambda}$ gives a type IIB theory in which the $(p, q)$ string is now the fundamental string and $\Omega_{\Lambda}$ acts as parity reversal on the $(p, q)$ string world-sheet. Modding out by $\Omega_{\Lambda}$ should again give a type I string (for weak $g_{\Lambda}$ ) and a heterotic string (for strong $g_{\Lambda}$ ); each choice of $\Lambda$ gives a physically equivalent theory, but 'embedded' differently in the type IIB string. 


\section{The IIA Theory and M-Theory}

We have argued that there should be an extra 9-brane in the type IIB theory. Dimensionally reducing on a circle to 9 dimensions, the 9-brane yields a new 8brane in the $D=9$ type II theory, in addition to the D-8-brane that comes from the RR 9-brane in the IIB theory. The same $D=9$ type II theory can be obtained by reducing the T-dual type IIA theory, so that the new 8-brane should also have a type IIA origin. It could have come from either a new 8-brane or a new 9-brane in the $D=10$ type IIA theory; however, a new type IIA 8-brane would give both an 8-brane and a 7-brane in $D=9$, and there is no evidence for an extra 7-brane from the type IIB side. Thus the type IIA theory should also have a new 9-brane, which again is not in the RR sector and is not a D-brane. The type IIA theory at finite coupling is M-theory compactified on a circle, so that the new type IIA 9-brane should have an M-theory origin. The simplest way in which this could happen would be if M-theory had either a 9-brane or a 10-brane. A 10-brane would give just a 9-brane in $D=10$, while a 9-brane would give a 9-brane and an 8-brane. We have seen in section 2 that the $D=11$ superalgebra (2.1) has a 9-brane charge that reduces to an 8-brane charge and a 9-brane charge in $D=10$, so we conclude that M-theory could have a 9-brane that gives rise to the new 9-brane and the 8-D-brane of type IIA on reducing on a circle.

Assuming the existence of an M-9-brane, M-theory then has $p$-branes for $p=$ $2,5,6,9$, and reducing to the $D=10$ IIA theory, the M-membrane gives branes with $p=1,2$, the M-5-brane gives branes with $p=4,5$, the M-6-brane gives branes with $p=5,6$ and the M-9-brane gives branes with $p=8,9$. In addition, the 11momentum $P_{M}$ gives a 0-brane charge and a 10-momentum on reduction. If the $D$-momentum $P_{M}$ were treated in the same way as the other charges, it would be split into the spatial momentum $P_{i}$ which is analogous to a 1-brane charge, and the energy $P_{0}$, which could be dualised to a $D-1$ brane charge $\hat{P}_{i_{1} \ldots i_{D-1}}$. The fact that states carrying momentum in compactified dimensions are U-dual to states obtained by wrapping branes round the compactified dimensions [2] means that 
for some purposes states carrying the gravitational charge $P_{M}$ should be treated on the same footing as states carrying brane charges.

If the $D=11$ momentum $P_{M}$ is formally thought of as corresponding to a 1-brane charge $P_{i}$ and a 10-brane charge $\hat{P}_{i_{1} \ldots i_{D-1}}$ (dual to $P_{0}$ ), then the M-theory spectrum has branes with $p=1,2 ; 5,6 ; 9,10$. It is suggestive that these occur in three pairs $\{p-1, p\}$ for $p=2,6,10$. This would have a natural interpretation if these charges arose from a 12-dimensional theory with a 2-brane, a 6-brane and a 10-brane. Twelve-dimensional theories have been proposed for a number of different reasons $[7,47,48]$. If the 12 -dimensional space has signature $(11,1)$, then this reduction is straightforward. It has been suggested [47] that the superalgebra in $10+2$ dimensions [17]

$$
\left\{Q_{\alpha}, Q_{\beta}\right\}=\left(\Gamma^{M N} C\right)_{\alpha \beta} Z_{M N}+\left(\Gamma^{M N P Q R S} C\right)_{\alpha \beta} Z_{M N P Q R S}^{+}
$$

(with $Q_{\alpha}$ 32-component Majorana-Weyl spinors, and $Z_{M N P Q R S}^{+}$a self-dual 6-form) should play a role in 12 dimensions. On dimensional reduction with respect to one of the time-like directions, the $D=11$ algebra (2.1) emerges, with the momentum $P_{M}$ arising from the $D=12$ 2-form charge $Z_{M N}$. Choosing one of the two times $x^{0}$ as the 'canonical time', the remaining coordinates are the other time coordinate $x^{\hat{0}}$ and the spatial coordinates $x^{i}(i=1, \ldots, 10)$, which can be combined into 11dimensional coordinates $x^{\mu}$, with $\mu=\hat{0}, i$. The 2 charges $Z_{M N}, Z_{M N P Q R S}^{+}$give rise to a 2 -brane charge $Z_{\mu \nu}$ and a 6 -brane charge $Z_{\mu_{1} \ldots \mu_{6}}$, while $Z_{0 \mu}$ is dualised to give a 10-brane charge $\hat{Z}_{\mu_{1} \ldots \mu_{10}}$; the dual 6-brane charge $\hat{Z}_{\mu_{1} \ldots \mu_{6}}$ is equal to $Z_{\mu_{1} \ldots \mu_{6}}$ because of the self-duality of $Z_{M N P Q R S}^{+}$. Each of these $p$-brane charges with $p=2,6,10$ splits into one including an $x^{\hat{0}}$ component and one without, giving the charge for a brane with world-volume of signature $(p, 1)$ and one for a brane with world-volume of signature $(p-1,2)$. On dimensionally reducing in the $x^{\hat{0}}$ direction, one obtains in 11 dimensions a $p$-brane and a $p-1$ brane, both with conventional Lorentzian signature. 


\begin{tabular}{|c|c|c|c|}
\hline 12-Brane & World-Volume Signature & Charge & M-brane \\
\hline 2-brane & $(2,1)$ & $Z_{i j}$ & 2 -brane \\
\hline$(1,1)$-brane & $(1,2)$ & $Z_{i \hat{0}}$ & pp-wave \\
\hline 6-brane & $(6,1)$ & $Z_{i j k l m n}$ & KK monopole \\
\hline$(5,1)$-brane & $(5,2)$ & $Z_{i j k l m \hat{0}}$ & 5 -brane \\
\hline 10 -brane & $(10,1)$ & $Z_{i_{1} \ldots i_{10}}$ & Energy $P_{0}$ \\
\hline$(9,1)$-brane & $(9,2)$ & $Z_{i_{1} \ldots i_{0} \hat{0}}$ & 9 -brane \\
\hline
\end{tabular}

Table 1 Suggested branes in 12 dimensions and reduction to 11 dimensions.

The conventional type IIA supergravity has no 8-brane solution, but it was shown in [24] that Romans' generalisation of the type IIA theory involving a mass $m$ [49] does have such a solution. The 8-brane couples to a non-dynamical 9-form potential $A_{9}$ with field strength $F_{10}$. The massive type IIA action was rewritten in $[24]$ in a form which included the term

$$
\int M F_{10}
$$

where $M$ is a scalar field. As $A_{9}$ only occurs in the action through this term, it is a Lagrange multiplier imposing the constraint $M=$ constant and the constant value of $M$ is the mass $m$ of the Romans theory. If $m=0$, the standard type IIA supergravity is recovered. This 8-brane should arise from the double dimensional reduction of the 9-brane of M-theory, and the term (7.2) should also have an 11dimensional origin. Indeed, it was argued in [24] that the term (7.2) necessarily arises in the IIA string theory, which implies that a similar term must arise in Mtheory, as the M-theory compactified on a circle is the same thing as the type IIA string at finite coupling. This suggests that the low-energy effective field theory of M-theory should include a term

$$
\int M F_{11}
$$

where $M$ is a scalar and $F_{11}=d A_{10}$ where $A_{10}$ is a 10 -form potential which couples to the M-theory 9-brane. On reduction to $D=10$, the potential $A_{10}$ gives $A_{9}$ which couples to the IIA 8-brane, and a 10-form $A_{10}$ which couples to the 9brane. However, it has been shown subject to certain assumptions that there is no 
conventional extension of $D=11$ supergravity which includes a term of the form (7.3) [59], so that any modification of the supergravity theory including the term (7.3) must be of an unusual form.

\section{World-Volume Theories and Collective Coordinates}

The zero-mode dynamics of a $p$-brane are described by a supersymmetric field theory on the $p+1$ dimensional world-volume arising from gauge-fixing a $\kappa$-symmetric supercovariant action. This world-volume theory has 8 fermionic and 8 bosonic degrees of freedom for branes that break half the supersymmetry. The bosonic degrees of freedom for a $p$-brane usually include $D-p-1$ scalar fields, corresponding to the collective coordinates for the position of the $p$-brane, and if $D-p-1<8$, there are in addition $9+p-D$ bosonic degrees of freedom which are typically collective coordinates for anti-symmetric tensor gauge field degrees of freedom. The $D=10$ fundamental string is described by 8 world-sheet scalars and 8 world-sheet spinors, while the $D=11$ membrane is described by 8 worldvolume scalars and 8 3-dimensional spinors; the scalars transform as a vector and the fermions as a spinor under the transverse $S O(8)$. A D-brane is described by a vector multiplet in its $p+1$ dimensional world-volume with $9-p$ scalars and a vector field, giving $(9-p)+(p-1)=8$ bosonic physical degrees of freedom. The 5-brane of M-theory is described by a 6-dimensional self-dual vector multiplet whose bosonic degrees of freedom consist of 5 scalars and a 2-form gauge field with self-dual field strength.

The description of the dynamics of the membrane in $D=11$ requires 8 scalars, while the $D=10$ membrane of the type IIA theory should have one less scalar collective coordinate; this is indeed the case, and its effective world-volume theory has 7 scalars and a vector. In the three-dimensional world-volume, a scalar is dual to a vector, and the two membrane effective actions are related by a world-

volume duality transformation. Similarly, the type IIA 5-brane should have 4 scalars on its world-volume, while the M-theory 5-brane from which it is obtained 
by double dimensional reduction has 5 scalars. This suggests that the IIA 5 -brane should be described by the 6-dimensional multiplet obtained by taking the selfdual antisymmetric tensor multiplet and dualising one of the scalars to obtain a 4 -form. The scalar to be dualised is the one corresponding to translations in the 11-th dimension, which is taken to be an isometry in the dimensional reduction, so that the scalar only appears through its derivative and can be dualised. Thus the bosonic sector of the M-theory 5-brane effective theory has 5 scalars and a self-dual 2-form gauge field, the IIA 5-brane has 4 scalars, a self-dual 2-form gauge field and a 4-form gauge field; the two multiplets are on-shell equivalent. In the IIB theory, the D-5-brane is described by a vector multiplet with 4 scalars and a vector. This can be dualised to a multiplet with 4 scalars and a 3-form gauge field, and this is a natural candidate for the world-volume theory of the NS 5-brane of the type IIB theory.

In $[48,50]$, explicit duality transformations were considered for D-brane actions, and for the cases considered the results were consistent with the view that the transition from weak to strong coupling is accompanied by a world-volume duality transformation. However, the determination of the zero modes from the supergravity field equations, as in [20], does not distinguish between dual forms of the world-volume collective coordinate supermultiplet; the choice between dual forms of the multiplets seems to be a matter of convenience rather than of principle. For example, for the membrane the formulation with eight scalars makes the (transverse part of the) 11-dimensional Lorentz invariance manifest, while it is more convenient to write the non-abelian generalisation arising when a number of membranes are coincident in terms of the vector multiplet, as the corresponding action written in terms of the scalars would be non-local. In what follows, we shall seek dual forms of the multiplet in which the number of scalars is the number of translational zero-modes, and different branes have different multiplets.

We now turn to the question of the KK monopoles. We have argued that they can be regarded as $D-5$ branes, which should imply that they have 4 scalar degrees of freedom (since a $p$-brane should have $D-p-1$ scalar fields). However, this 
cannot be correct, as this number of scalars cannot be fitted into supermultiplets of half-maximal supersymmetry in general. For example, for $D=11$ we would need a 7-dimensional $N=2$ supermultiplet with 4 scalars, of which none are known. In 7 dimensions, an $N=2$ vector multiplet has three scalars and a vector, and the number of scalars can be decreased by dualising, but cannot be increased. The resolution comes from considering the gravitational instanton moduli space. The space-time in question is $\mathbb{R}^{p, 1} \times N_{4}$ where $N_{4}$ is a gravitational multi-instanton space. For a single instanton in four dimensions, one would expect four collective coordinates corresponding to the position of the instanton. However, this is not the case for the Taub-NUT metric, which has a 3-dimensional moduli space; translation in the direction $\partial / \partial y$ gives an equivalent metric and so does not count as a deformation as that direction is Killing [51]. More generally, the multi-centre metrics (5.2),(5.26),(5.27), have only $3 s$ moduli (corresponding to the $s$ positions $x_{i}^{\mu}$ in $\mathbb{R}^{3}$ ) [51]. This would be consistent with the effective world-volume dynamics of the KK monopole being described by a 7-dimensional vector multiplet, which has 3 scalars, or by the multiplet given by dualising the vector to give 3 scalars and a 4-form gauge field; these are the only possibilities as no other matter multiplets have 3 scalars. It is remarkable that supersymmetry would have led us to the conclusion that the moduli space dimension should be a multiple of 3 .

Double dimensional reduction to the KK monopole 5-brane in 10 dimensions gives the dimensional reduction of the vector multiplet to $D=6$, and should contain 3 scalars again, corresponding to the instanton moduli. This leads to the multiplet with 3 scalars, one 3-form and one 4-form gauge field, coming from the reduction of the multiplet with 3 scalars and a 4 -form in 7 dimensions. This suggests that a convenient description is in terms of a multiplet with 3 scalars and a 4-form in 7 dimensions or the multiplet obtained by straightforward dimensional reduction of this to 7 - $\mathrm{d}$ dimensions for the $\mathrm{KK}$ monopole in $D-d$ dimensions.

Simple dimensional reduction of the KK monopole (taking a periodic array) gives the type IIA 6 -brane in $D=10$. The 7 -dimensional world-volume description is obtained by dualising the 4 -form to a vector to give the $D=7$ vector multiplet 
with 3 scalars and a vector. The picture that seems to be emerging is that double dimensional reduction of an M-theory $p$-brane for $p=2,5,6$ gives a $p-1$ brane of the IIA theory with the world-volume theory obtained by dimensional reduction of the M-brane world-volume theory from $p+1$ to $p$ dimensions. On the other hand, simple dimensional reduction (with a periodic array) to give a IIA $p$-brane gives equivalent world-volume theories for the IIA and M-theory $p$-branes, but related by a world-volume duality transformation. If this pattern persists for the M-theory 9-brane, its world-volume theory must be a 10-dimensional supersymmetric theory whose dimensional reduction to 9 dimensions gives the 8-brane world-volume theory which is given by a vector multiplet with a vector and a scalar. This fixes the M-theory 9-brane to be described by a 10-dimensional vector multiplet. One would have expected a scalar representing the position of the 9-brane, but perhaps this is missing for reasons similar to those for the absence of the expected fourth modulus for the Kaluza-Klein monopole. Then the world-volume theory for the IIA 9-brane should be the multiplet related to this by a world-volume duality transformation, with a 7 -form gauge potential instead of a vector.

Consider now the IIB theory. As the solitonic (NS) 5-brane is related to the Kaluza-Klein monopole by T-duality (with respect to the isometry generated by $\partial / \partial y$ for the metric (5.2)), the IIA theory in a 5-brane background is equivalent to the IIB theory in a KK monopole background, and the IIB theory in a solitonic 5-brane background is equivalent to the IIA theory in a KK monopole background. This fixes the world-volume theory of the IIB KK monopole to be the six dimensional $(2,0)$ supermultiplet with a 2 -form $A_{2}^{+}$with self-dual field strength and 5 scalars, or a dual version of this. As there are 3 translational zero modes, it is natural to seek a dual form with 3 scalars; if it is possible to dualise 2 scalars, this would give a multiplet with $A_{2}^{+}, 3$ scalars and two 4 -form potentials $A_{4}$. (Note that the five scalars could have interpretation as translational zero modes of 7 -brane in 12 dimensions which reduces to this 5-brane in 10 dimensions.) It also fixes the six-dimensional world-volume multiplet for the type IIB NS-NS 5-brane to be the $(1,1)$ vector supermultiplet with 4 scalars and a vector, or a dual version of this. 
There should be 4 translational zero modes and so 4 scalars, but the vector could be dualised to give a multiplet with 4 scalars and a 3 -form.

Finally, we consider the pp-wave solutions. As there is a sense in which they can be regarded as 1-branes and are T-dual to fundamental strings, we can ask what their world-sheet effective dynamics should be. The pp-wave solution of Mtheory [52] gives the 0-brane of the IIA theory on double dimensional reduction (i.e. on reducing the configuration in which the wave travels in the compact 11th dimension) [3] and as the 0-brane world-line theory is a one-dimensional supervector multiplet with a vector gauge field and 9 scalars, the pp-wave world-sheet theory which reduces to this must be a 2-dimensional theory with 8 scalars and a vector. If the pp-wave is moving in a compact dimension, then it is related by T-duality to a fundamental string wrapped around the compact dimension [16]. Thus the IIA theory in such a pp-wave background is T-dual to the IIB theory in a fundamental string background, i.e. with a fundamental string wrapped around the internal dimension [53]. This fundamental string is in turn dual to a D-string. The effective world-sheet dynamics is then described by a IIB Green-Schwarz string with $8+8$ degrees of freedom in static gauge, or equivalently by a Born-Infeld action which is equivalent to this via a world-sheet duality transformation [50]. Similarly, the IIB pp-wave is T-dual to the IIA fundamental string [53], and is described by a IIA Green-Schwarz superstring action.

These world-volume dynamics are summarised in the following tables. The bosonic fields in the supermultiplet are listed, with $A_{n}$ denoting an $n$-form gauge potential, $A_{2}^{+}$denoting a 2 -form with self-dual field strength and $m \times \phi$ represents $m$ scalars. The multiplets for the solitonic 5-branes have also been proposed in $[55]$. 


\begin{tabular}{|c|c|c|}
\hline M-Brane & World-Volume Multiplet & Suggested Dual Form \\
\hline pp-Wave & $A_{1}, 8 \times \phi$ & $A_{1}, 8 \times \phi$ \\
\hline 2-Brane & $8 \times \phi$ & $8 \times \phi$ \\
\hline 5-Brane & $A_{2}^{+}, 5 \times \phi$ & $A_{2}^{+}, 5 \times \phi$ \\
\hline 6-Brane (KK Monopole) & $A_{1}, 3 \times \phi$ & $A_{4}, 3 \times \phi$ \\
\hline 9-Brane & $A_{1}$ & $A_{1}$ \\
\hline
\end{tabular}

Table 2 World-Volume Dynamics of M-Branes.

\begin{tabular}{|c|c|c|}
\hline IIA-Brane & World-Volume Multiplet & Suggested Dual Form \\
\hline $\begin{array}{c}\text { Dirichlet p-Brane } \\
\mathrm{p}=0,2,4,6,8\end{array}$ & $A_{1},(9-p) \times \phi$ & $A_{1},(9-p) \times \phi$ \\
\hline 1-Brane (Fundamental) & $8 \times \phi$ & $8 \times \phi$ \\
\hline pp-Wave & $A_{1}, 8 \times \phi$ & $A_{1}, 8 \times \phi$ \\
\hline 5-Brane (Solitonic) & $A_{2}^{+}, 5 \times \phi$ & $A_{2}^{+}, A_{4}, 4 \times \phi$ \\
\hline 5-Brane (KK Monopole) & $A_{1}, 4 \times \phi$ & $A_{4}, A_{3}, 3 \times \phi$ \\
\hline 9-Brane (Extra) & $A_{1}$ & $A_{7}$ \\
\hline
\end{tabular}

Table 3 World-Volume Dynamics of Type IIA Branes.

\begin{tabular}{|c|c|c|}
\hline IIB-Brane & World-Volume Multiplet & Suggested Dual Form \\
\hline $\begin{array}{c}\text { Dirichlet p-Brane } \\
\mathrm{p}=1,3,5,7,9\end{array}$ & $A_{1},(9-p) \times \phi$ & $A_{1},(9-p) \times \phi$ \\
\hline 1-Brane (Fundamental) & $8 \times \phi$ & $8 \times \phi$ \\
\hline pp-Wave & $8 \times \phi$ & $8 \times \phi$ \\
\hline 5-Brane (Solitonic) & $A_{1}, 4 \times \phi$ & $A_{3}, 4 \times \phi$ \\
\hline 5-Brane (KK Monopole) & $A_{2}^{+}, 5 \times \phi$ & $A_{2}^{+}, 3 \times \phi, 2 \times A_{4}$ \\
\hline 9-Brane (Extra) & $A_{1}$ & $A_{7}$ \\
\hline
\end{tabular}

Table 4 World-Volume Dynamics of Type IIB Branes.

In the last section, the possibility that the branes of M-theory could have a 12-dimensional origin was discussed. If it is indeed the case that there is a 2brane, 6-brane and 10-brane in 12 dimensions which reduce to the M-branes, it is interesting to ask whether consistent world-volume dynamics could be attributed to them which gives the correct dynamics on dimensional reduction. The 2-brane in 12 dimensions should give rise to the 2-brane and pp-wave in 11 dimensions, both of which have 8 scalars, and so should itself have 8 scalars; this is one less than would have been expected for a standard 2-brane in 12 dimensions. The 6-brane is 
more problematic. On reduction to M-theory, it should give both the 5 brane with a 6-dimensional self-dual tensor multiplet and the 6 brane with a 7-dimensional vector multiplet. If the $D=12$ signature is $(11,1)$, the 6 -brane world-volume has signature $(6,1)$ and there is no 7-dimensional multiplet that is equivalent to the vector multiplet and reduces to the tensor multiplet. If the $D=12$ signature is (10,2), the M-theory 6-brane arises from the $D=12$ 6-brane with world-volume signature $(6,1)$, and this should then have a world-volume vector multiplet. The Mtheory 5-brane arises from the $D=12$-brane with world-volume signature $(5,2)$, and this should be described by a multiplet in $(5,2)$ dimensions that reduces to the self-dual tensor multiplet in $(5,1)$ dimensions. In particular, chiral fermions and a self-dual tensor must emerge on dimensional reduction, and this could not happen if the $(5,2)$ theory was a conventional local theory with $O(5,2)$ Lorentz invariance and with a standard dimensional reduction. Thus it appears that either the $D=12$ branes cannot have covariant dynamics - it might involve a particular vector, such as a fixed null vector - or the reduction to $D=11$ must be non-standard.

\section{ALE Branes, ALF Branes and Symmetry Enhancement}

When parallel D-branes of the same type approach one another, the worldvolume gauge symmetry is enhanced to a non-abelian group with extra massless states arising from fundamental strings joining the D-branes. The symmetry enhancement should be true for all values of the coupling, although the interpretation can change. The type IIA string becomes 11-dimensional M-theory at strong coupling, and D-branes for $p=0,2,4,5,6,8$ become, as we have seen, pp-waves, 2-branes, 5-branes, KK monopoles and 9-branes respectively, which can be thought of as M-branes with $p=1,2,5,6,9$. The configuration of two $p$-D-branes joined by fundamental strings, for which the world-volume theory has a $U(2)$ gauge symmetry when the branes coincide, becomes two M-branes joined by a 2-brane in $D=11$. Indeed, it was shown in [54] (and references therein) that an M-theory 2-brane can intersect a pp-wave, 2-brane, 5-brane or KK monopole in such a way 
that breaks $1 / 2$ the supersymmetry and which reduces to a fundamental string intersecting a D-brane with $p=0,2,4,6$ respectively. Then two parallel M-branes of the same type (pp-waves, 2-branes, 5-branes, KK monopoles or 9-branes) give enhanced symmetry when they come together, giving a non-abelian gauge theory or, in the case of 5-branes, a theory of tensionless self-dual strings [56]. As a result, in the IIA theory two fundamental strings, two pp-waves, two 5-branes or KK monopoles coming together gives enhanced symmetry. For the type IIB theory, enhanced symmetry also results from bringing together two fundamental strings, two solitonic 5 -branes or two (1,0) 9-branes, as these are related by $S L(2, \mathbb{Z})$ duality to D-branes, and from bringing together two KK monopoles or two pp-waves, as these are related by T-duality to type IIA 5-branes and fundamental strings, respectively. We now examine this in more detail for the case of KK monopoles.

The Gibbons-Hawking gravitational multi-instanton metric $[28,57]$ is

$$
d s^{2}=V\left(d y+A_{\mu} d x^{\mu}\right)^{2}+V^{-1} \delta_{\mu \nu} d x^{\mu} d x^{\nu}
$$

where

$$
V^{-1}=\epsilon+\sum_{i=1}^{s} \frac{2 n}{\left|x^{\mu}-x_{i}^{\mu}\right|}, \quad F_{\mu \nu}=\epsilon_{\mu \nu \rho} \nabla^{\rho} V^{-1}
$$

If $\epsilon=1$, this is the multi-Taub-NUT space considered earlier with ALF (asymptotically locally flat) boundary conditions. If $\epsilon=0, n$ can be scaled to 1 and the space is a multi-centre generalisation of the Eguchi-Hanson metric with ALE (asymptotically locally Euclidean) boundary conditions. The case $\epsilon=0, s=1$ is flat space, while $\epsilon=0, s=2$ gives the Eguchi-Hanson instanton. The space $\mathbb{R}^{D-5,1} \times N_{4}$ where $N_{4}$ is an ALF or ALE instanton is a solution of M-theory $(D=11)$ or of string theory $(D=10)$ as the gravitational instanton is hyperkahler [29].

The multi-Eguchi-Hanson space behaves as $\mathbb{R}^{4} / \mathbb{Z}_{s}$ at large distances, and can be used to resolve an $A_{s}$ singularity of an orbifold limit of $K_{3}$, or blow up the

\footnotetext{
$\star$ Some of the following was also considered in [60].
} 
orbifold singularity of $\mathbb{R}^{4} / \mathbb{Z}_{s}$. For example, one orbifold limit of $K_{3}$ is $T^{4} / \mathbb{Z}_{2}$ and each of the 16 orbifold singularities can be repaired by gluing in an Eguchi-Hanson metric. In the limit $\left|x_{1}-x_{2}\right| \rightarrow 0$, the Eguchi-Hanson space becomes $\mathbb{R}^{4} / \mathbb{Z}_{2}$ with an orbifold singularity at $x_{1}$. While the multi-Taub-NUT solution can be thought of as a solution with a number of parallel $D-5$ G-branes, the multiEguchi-Hanson space can be viewed as a number of parallel $D-5$ G-branes in a transverse space which is an orbifold; as a result, not all the branes are independent as some are 'mirror images' of others. There is one instanton corresponding to each pair $\left\{x_{i}, x_{j}\right\}$.

As the $y$ coordinate is periodic, each line segment in the $\mathbb{R}^{3}$ parameterised by $x^{\mu}$ is associated with a cylinder in $N_{4}$, unless the line segment passes through one of the points $x_{i}^{\mu}$ at which the size of the $y$-circle shrinks to zero. In particular, a line segment joining $x_{i}^{\mu}$ and $x_{j}^{\mu}$ corresponds to a 2-sphere and the set of all such 2-spheres corresponding to all pairs $\left\{x_{i}, x_{j}\right\}$ forms a basis for the second homology. As one approaches a point in moduli space at which $\left|x_{i}-x_{j}\right| \rightarrow 0$, the area of the corresponding 2-sphere shrinks to zero and this is associated with symmetry enhancement.

For type IIA string theory compactified on $K_{3}$, the equivalence with heterotic string theory implies the existence of special points in the $K_{3}$ moduli space at which the gauge symmetry is enhanced [2]. Indeed, it follows from supersymmetry that the mass of certain BPS states tends to zero at these special points $[6,8]$ to give the extra massless vector multiplets. At these points the area of certain homology 2-cycles shrinks to zero to give an orbifold limit of $K_{3}$ and the BPS states in 6 dimensions arising from 2-branes wrapped around the shrinking 2-cycles become massless $[4,6,8]$. The behaviour of the theory as a particular set of 2-cycles shrinks can be studied by looking at the theory on the space $\mathbb{R}^{D-5,1} \times N_{4}$ in which the $K_{3}$ is replaced by the appropriate multi-Eguchi-Hanson space [58]; this gives a good approximation when the radius of the 2-sphere is small compared to the size of the $K_{3}$. The enhanced gauge symmetry is then associated with parallel G-branes becoming coincident, so that homology 2-spheres shrink to zero size and 
the membranes wrapped around these give rise to massless states on the G-brane so that the G-brane world-volume theory becomes a non-abelian gauge theory. (The world-volume theory for each G-brane is a $D=6$ vector multiplet, as for the ALF case.) This is straightforward to check using duality; a T-duality relates the solution to a multi-centre solitonic 5 -brane solution of type IIB theory (in $\mathbb{R}^{4} / \mathbb{Z}_{s}$ ) and $S L(2, \mathbb{Z})$ duality relates this to a configuration of parallel 5-D-branes, and there is symmetry enhancement as these become coincident due to strings joining the 5-D-branes becoming massless [58]. In the case of the Eguchi-Hanson space $(s=2)$, the instanton shrinks to zero size as the 2-sphere shrinks, so that the symmetry enhancement is a gravitational analogue of the symmetry enhancement as certain Yang-Mills instantons shrink to zero size [61].

There is a similar symmetry enhancement as KK monopole G-branes become coincident. This is to be expected from the fact that the 6-D-brane of type IIA arises from the KK monopole of M-theory [3]; the symmetry enhancement of the world-volume theory as the 6-D-branes become coincident implies the same enhancement as parallel KK monopole G-branes of M-theory become coincident, and this in turn implies a similar effect for KK monopoles of the type IIA theory. For the 6-D-brane, the extra massless states come from strings joining the approaching 6-D-branes, while for the KK monopole $D-5$ G-branes, these come from membranes wrapping around the shrinking 2-cycles. The position of each $D-5 \mathrm{G}$-brane corresponds to a point $x_{i}$ in $\mathbb{R}^{3}$ and there is a 2 -sphere for each pair $\left\{x_{i}, x_{j}\right\}$. Thus when two parallel G-6-branes of M-theory approach one another, the $D=7 U(1)$ vector supermultiplet on each brane combines with the BPS state from membranes wrapping the 2-cycle joining them to give a $U(2)$ super-Yang-Mills theory on the common 7-dimensional world-volume. This enhancement can be understood in the IIA case from duality using the argument of [58]; the two parallel G-5-branes of the IIA theory are T-dual to two parallel solitonic 5-branes of the IIB theory, and these are $S L(2, \mathbb{Z})$-dual to two parallel 5-D-branes, with the usual $U(1) \times U(1) \rightarrow U(2)$ symmetry enhancement on the world-volume. The membrane wrapping the 2-cycle is replaced by the string joining the D-branes. 
Consider now the analogous situation in the IIB string on $\mathbb{R}^{5,1} \times K_{3}$ or $\mathbb{R}^{5,1} \times N_{4}$. In this case, three branes wrapping the 2-cycles gives rise to self-dual strings in 6 dimensions that become tensionless in the limit in which the 2-cycle shrinks to zero area [9]. In particular, the limit in which two parallel G-5-branes become coincident, the world-volume theory changes from an abelian self-dual anti-symmetric tensor multiplet to a non-abelian generalisation resulting from self-dual strings becoming null. This is related by T-duality to the case of 2 parallel 5 -branes of the IIA theory becoming coincident, which in turn becomes the case of coincident 5-branes of M-theory at strong coupling; in this latter case, the 3-brane wrapping the 2-cycle have been replaced by a membrane ending on the two 5-branes [56].

Acknowledgements: I would like to thank Jerome Gauntlett, Gary Gibbons, Michael Green, Ashoke Sen and Paul Townsend for valuable discussions.

\section{REFERENCES}

1. A. Sen, Nucl. Phys. B404 (1993) 109; Phys. Lett. 303B (1993); Int. J. Mod. Phys. A8 (1993) 5079; Mod. Phys. Lett. A8 (1993) 2023; Int. J. Mod. Phys. A9 (1994) 3707.

2. C.M. Hull and P.K. Townsend, Nucl. Phys. B438 (1995) 109.

3. P.K. Townsend, Phys. Lett. B350 (1995) 184.

4. E. Witten, Nucl. Phys. B443 (1995) 85, hep-th/9503124.

5. P.K. Townsend, in the Proceedings of the March 95 PASCOS/John Hopkins Conference, hep-th/9507048.

6. C.M. Hull, in Proceedings of Strings 95.

7. C.M. Hull, Nucl. Phys. B468 (1996) 113, hep-th/9512181.

8. C.M. Hull and P.K. Townsend, Nucl. Phys. B451 (1995) 525, hepth/9505073. 
9. E. Witten, in Proceedings of Strings 95, hep-th/9507121.

10. C.M.Hull, Phys. Lett. B357 (1995) 545, hep-th/9506194.

11. A. Dabholkar, Phys. Lett. B357 (1995) 307, hep-th/9506160.

12. A. Sagnotti, in "Non-Perturbative Quantum Field Theory", Proceedings of 1987 Cargese Summer Institute, eds. G. Mack et al (Pergamon, 1988), p. 521.

13. J. Polchinski and E. Witten, Nucl. Phys. B460 (1996) 525.

14. R. Sorkin, Phys. Rev. Lett. 51 (1983) 87 ; D. Gross and M. Perry, Nucl. Phys. B226 (1983) 29.

15. G.W. Gibbons and C.M. Hull, Phys. Lett. 109B (1982) 190.

16. E. Bergsoeff, I. Entrop and R. Kallosh, Phys. Rev. D 49 (1994) 6663.

17. J. W. van Holten and A. Van Proeyen Phys. A: Math. Gen. 15 (1982) 3763.

18. A. Dabholkar, G.W. Gibbons, J.A. Harvey and F. Ruiz-Ruiz, Nucl. Phys. B340 (1990) 33.

19. G.T. Horowitz and A. Strominger, Nucl. Phys. B360 (1991) 197.

20. C. Callan, J. Harvey and A. Strominger, Nucl. Phys. B359 (1991) 611.

21. M.J. Duff and J.X. Lu, Nucl. Phys. B354 (1991) 141; Phys. Rev. Lett. 66 (1991) 1402; Phys. Lett. 273B (1991) 409; M.J. Duff, G.W. Gibbons and P.K. Townsend, Phys. Lett. 332B (1994) 321.

22. M.J. Duff, Class. Quant. Grav. 5 (1988) 189; A. Strominger, Nucl. Phys. B343 (1990) 167; M.J. Duff and J.X. Lu, Nucl. Phys. B354 (1991) 129 and Nucl. Phys. B357 (1991) 534.

23. G.W. Gibbons, M.B. Green and M.J. Perry, Phys. Lett. B370 (1996) 37.

24. E. Bergshoeff, M. de Roo, M.B. Green, G. Papadopoulos and P.K. Townsend, Nucl. Phys.B470 1996113.

25. E. Witten, Nucl. Phys. B460 1996335. 
26. M. Douglas and M. Li, hep-th/9604041.

27. B.A. Ovrut and D. Waldram, hep-th/9704045.

28. S.W. Hawking, Phys. Lett. 60A (1977) 81.

29. C.M. Hull, Nucl. Phys. B260 (1985) 182 ; L. Alvarez-Gaumé and P. Ginsparg, Commun. Math. Phys. 102 (1985) 311.

30. S. Ramaswamy and A. Sen, J. Math. Phys. 22 (1981) 2612.

31. A. Ashtekar and A. Sen, J. Math. Phys. 23 (1982) 2168.

32. J.M. Nester, Phys. Lett. 83A (1981) 241.

33. C.M. Hull, Commun. Math. Phys. 90 (1983) 545.

34. Gibbons G W, Horowitz G T and Townsend P K, Class. Quantum Grav. 12 (1995) 297.

35. E.Witten, Commun. Math. Phys. 80 (1981) 381.

36. T. Parker and C. Taubes, Commun. Math. Phys. 84 (1982) 223; O. Reula, J. Math. Phys. 23(1982) 5.

37. G.W. Gibbons and M.J. Perry, Nucl. Phys. B248 (1984) 629.

38. J. Lee and R.D. Sorkin, Commun. Math. Phys. 116 (1988) 353.

39. O.M. Moreschi and G.A.J. Sparling, J. Math. Phs. 27 (1986) 2402.

40. L. Bombelli, R.K. Koul, G. Kunstatter, J. Lee and R.D. Sorkin, Nucl. Phys. B289 (1987) 735.

41. B. Jensen and P.V. Mani, hep-th/9702044.

42. R. Kallosh, D. Kastor, T. Ortin and T. Torma, Phys. Rev. D50 (1944) 6374.

43. E. Witten, Nucl. Phys. B289 (1982) 735.

44. M.M. Taylor-Robinson, hep-th/9609234.

45. G.W. Gibbons and S.W. Hawking, Commun. Math. Phys. 61 (1979) 291. 
46. A.Sen, Nucl. Phys. B474 (1996) 361, hep-th/9604070; Nucl. Phys. B475 (1996) 562, hep-th/9605150; hep-th/9609176

47. M. Blencowe, M.J. Duff, C.M. Hull and K.S. Stelle, unpublished; M. Blencowe and M.J. Duff, Nucl. Phys. 310 (1988) 387; D. Kutasov and E. Martinec, hep-th/9510182; D. Kutasov, E. Martinec and M. O'Loughlin, hep-th/9603116; I. Bars, hep-th/9604200.

48. A.A. Tseytlin, Nucl. Phys. B469 (1996) 51.

49. L. Romans, Phys. Lett. 169B (1986) 374.

50. M. Aganagic, J. Park, C. Popescu and J. Schwarz, hep-th/9702133; M. Abou Zeid and C.M. Hull, hep-th/9704021; M.B. Green and M. Gutperle, Phys. Lett. B377 (1996) 28; Y. Lozano, hep-th/9701186.

51. P. Ruback, Commun. Math. Phys. 107 (1986) 93.

52. C.M. Hull, Phys. Lett. B139 (1984) 39.

53. E. Bergsoeff, C.M. Hull and T. Ortin, Nucl. Phys. 451 (1995) 547.

54. E. Bergshoeff, M. de Roo, E. Eyras, B. Janssen and J.P. van de Schaar, hep-th/9704120.

55. E. Bergshoeff, hep-th/9607238, 9611099.

56. A. Strominger, Phys. Lett. B383 (1996) 44.

57. G.W. Gibbons and S.W. Hawking, Phys. Lett. 78B (1978) 430.

58. M. Berschadsky, V. Sadov and C. Vafa, Nucl. Phys. B463 (1996) 398, hep-th/9510225.

59. K. Bautier, S. Deser, M. Henneaux and D. Seminara, hep-th/9704131.

60. P.K. Townsend, lectures at the Euroconference: Duality and Supersymmetric Theories, Newton Institute, 1997.

61. E. Witten, Nucl. Phys. B460 (1996) 541. 